\title{
Stimulated Raman adiabatic passage in the presence of dephasing
}

\author{
Qiang Shi and Eitan Geva ${ }^{\text {a) }}$ \\ Department of Chemistry and The FOCUS Center, University of Michigan, \\ Ann Arbor, Michigan 48109-1055
}

(Received 25 June 2003; accepted 12 September 2003)

\begin{abstract}
The prospect of employing the stimulated Raman adiabatic Passage (STIRAP) technique under the influence of pure dephasing is explored. A general analysis of how decoherence influences the performance of STIRAP is provided. Starting from a general and fully quantum-mechanical system-bath Hamiltonian, we derive a quantum master equation (QME) that describes the reduced dynamics of a dissipative STIRAP system. The derivation is based on the standard assumptions of (1) weak system-bath coupling; (2) Markovity, in the sense that the relaxation times are long in comparison to the bath correlation time, $\tau_{c}$; and (3) weak field-matter interaction, in the sense that the Rabi period of the driving laser fields, $\Omega^{-1}$, is longer than $\tau_{c}$. The dissipative term in this QME is the same as it would have been in the absence of the driving fields, because of the assumption of weak field-matter interaction. This type of uncontrollable dephasing is seen to diminish the efficiency of STIRAP, although the actual loss strongly depends on the specific dephasing mechanism. We also derive a more general QME, which is applicable to driving fields of arbitrary intensity. The dissipative term in the new QME is explicitly dependent on the driving fields, and therefore controllable. Intense fields are shown to effectively slow down the dephasing when $\Omega \tau_{c}>1$, which suggests that it may be possible to use STIRAP in order to transfer population between the quantum states of a solute molecule embedded in a solvent. (C) 2003 American Institute of Physics. [DOI: 10.1063/1.1623482]
\end{abstract}

\section{INTRODUCTION}

The goal of quantum coherent control is the design of laser pulses for controlling molecular motion and chemical reactivity. ${ }^{1-6}$ The main question is formulated in terms of optimal control theory: Given the constraints on the duration and intensity of the pulse, what temporal changes of the amplitude and phase of the laser field would maximize a desired objective? The objective is usually represented by an expectation value at the final state. The optimal pulse is found by solving the optimal control problem, either computationally, via quantum molecular dynamics simulations ${ }^{7-9}$ or experimentally, via a closed adaptive learning loop. ${ }^{10-12}$

Although most of the studies to date have been focused on gas phase systems, there is a rapidly growing interest in exploring the prospects of coherently controlling processes in solution. ${ }^{13-31}$ This interest is fueled by the fact that many important chemical and physical processes that one would like to control take place in condensed phase solutions, as well as by the development of the closed loop self-adaptive learning approach, ${ }^{10-12,32-39}$ which made it possible to explore the prospects of controlling complex systems, without a detailed knowledge of the full Hamiltonian.

The theoretical analysis of coherent control in solution is significantly more demanding than its gas phase counterpart. More specifically, while gas-phase dynamics can usually be treated as decoherence-free, solution-phase dynamics are strongly influenced by relaxation processes, which reflect interactions with solvent degrees of freedom (DOF). Thus,

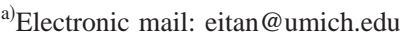

solution-phase dynamics often involves decoherence, which may suppress the very same coherences upon which coherent control is based (decoherence is defined here as any nonunitary contribution to the dynamics of the system). Thus, understanding the interplay between coherent control and decoherence, as well as exploring ways of controlling the destructive attributes of decoherence, is of paramount importance. Dephasing (phase relaxation), in particular, is often conceived as destructive for coherent control, as well as being uncontrollable. However, one of us has recently analyzed the control of laser-driven intramolecular hydrogen transfer in the presence of dephasing, ${ }^{29}$ and found that, at least for some types of dephasing mechanisms, dephasing can be effectively controlled, and even totally eliminated, by taking advantage of the field dependence of the dephasing rate constants. In the present paper, we explore the prospects of using the stimulated Raman adiabatic passage (STIRAP) technique $e^{40-50}$ in order to transfer population between two selected quantum states of a solute molecule, while the latter is subject to pure dephasing. We choose to focus on STIRAP because of its general applicability and the fact that it is based on a relatively simple sequence of two pulses, where the main control parameter is the time delay between them.

The plan of this paper is as follows: A brief description of dephasing-free STIRAP is given in Sec. II. A discussion of the influence of different types of relaxation processes on STIRAP is presented in Sec. III, with the conclusion that pure dephasing is the most important type of relaxation that needs to be considered. The influence of pure dephasing within the framework of standard quantum master equation (QME) theory, which is only valid at the limit of weak driv- 


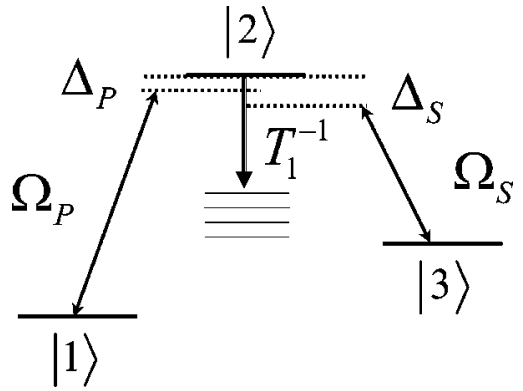

FIG. 1. A schematic view of the STIRAP three-level system in the $\Lambda$ configuration.

ing fields, is analyzed in Sec. IV. The treatment is extended to driving fields of arbitrary intensity in Sec. V, where it is shown that the dephasing rate is explicitly dependent on the driving field, and can be controlled by it. The relationship of our results to other works that considered the influence of decoherence effects on STIRAP, and decoherence control in general, is discussed in Sec. VI. The main results are summarized and discussed in Sec. VII. The theoretical results necessary for deriving a QME, in the case of a driven system, are outlined in the Appendix.

\section{DECOHERENCE-FREE STIRAP}

In this section we provide a short overview of the standard decoherence-free treatment of STIRAP. Our goal is to introduce the notation that will be used throughout the remainder of this paper, as well as provide a reference point for the later analysis of STIRAP in the presence of dephasing.

We consider a three-level system in the $\Lambda$ configuration, as shown in Fig. 1. The field-free system Hamiltonian is given by

$$
\begin{aligned}
\hat{H}_{s}^{0} & =\hbar \omega_{1}|1\rangle\left\langle 1\left|+\hbar \omega_{2}\right| 2\right\rangle\left\langle 2\left|+\hbar \omega_{3}\right| 3\right\rangle\langle 3| \\
& \equiv \hbar \omega_{1} \hat{P}_{1}+\hbar \omega_{2} \hat{P}_{2}+\hbar \omega_{3} \hat{P}_{3},
\end{aligned}
$$

where, $\{|1\rangle,|2\rangle,|3\rangle\}$ and $\left\{\hbar \omega_{1}, \hbar \omega_{2}, \hbar \omega_{3}\right\}$ are obviously the eigenvectors and eigenvalues of $\hat{H}_{s}^{0}$, respectively, and $\hat{P}_{i}$ $=|i\rangle\langle i|(i=1,2,3)$. We assume that the $1 \leftrightarrow 2$ and $2 \leftrightarrow 3$ transitions are driven by classical, circularly polarized, coherent light pulses, which can be described by the following fieldmatter interaction term:

$$
\begin{aligned}
\hat{W}(t)= & \frac{1}{2} \mu_{12} \epsilon_{P}(t)\left[|2\rangle\left\langle 1\left|e^{-i \omega_{P} t}+\right| 1\right\rangle\langle 2| e^{i \omega_{P} t}\right] \\
& +\frac{1}{2} \mu_{23} \epsilon_{S}(t)\left[|2\rangle\left\langle 3\left|e^{-i \omega_{S} t}+\right| 3\right\rangle\langle 2| e^{i \omega_{S} t}\right] \\
\equiv & \frac{1}{2} \mu_{12} \epsilon_{P}(t)\left[\hat{P}_{21} e^{-i \omega_{P} t}+\hat{P}_{12} e^{i \omega_{P} t}\right] \\
& +\frac{1}{2} \mu_{23} \epsilon_{S}(t)\left[\hat{P}_{23} e^{-i \omega_{S} t}+\hat{P}_{32} e^{i \omega_{S} t}\right] .
\end{aligned}
$$

Here, $\mu_{12}, \epsilon_{P}(t)$ and $\omega_{P}\left[\mu_{23}, \epsilon_{S}(t)\right.$ and $\left.\omega_{S}\right]$ are the transition dipole moment, amplitude and frequency of the pump (Stokes) pulses, respectively, and $\hat{P}_{i j}=|i\rangle\langle j|(i, j=1,2,3)$ are the corresponding field-free creation and annihilation operators. It should be noted that the following analysis would also be applicable to noncircularly polarized driving fields, as long as the rotating-wave approximation (RWA) is valid.
In the absence of decoherence, the system's state can be described in terms of a state vector (in the Schrödinger picture), $|\psi(t)\rangle$, whose dynamics is dictated by the Schrödinger equation

$$
i \hbar \frac{d}{d t}|\psi(t)\rangle=\left[\hat{H}_{s}^{0}+\hat{W}(t)\right]|\psi(t)\rangle .
$$

In STIRAP, one subjects the system, whose state is $|1\rangle$ at $t$ $=0$, to a "counter-intuitive" pulse sequence, where the Stokes pulse precedes, and overlaps with, the pump pulse. This pulse sequence ideally results in a complete transfer of population to state $|3\rangle$, without ever populating state $|2\rangle$ in the process.

The underlying mechanism is best understood in terms of the rotating frame $(\mathrm{RF})$ picture. The state vector in the RF picture is defined by

$$
\left|\psi^{r}(t)\right\rangle=e^{i \omega_{P} t \hat{P}_{2}-i\left(\omega_{S}-\omega_{P}\right) t \hat{P}_{3}}|\psi(t)\rangle,
$$

and its dynamics is dictated by

$$
i \hbar \frac{d}{d t}\left|\psi^{r}(t)\right\rangle=\hat{H}_{s}^{r}\left|\psi^{r}(t)\right\rangle,
$$

where,

$$
\begin{aligned}
\hat{H}_{s}^{r}(t)= & \hbar\left\{\Delta_{P} \hat{P}_{2}^{r}+\left(\Delta_{P}-\Delta_{S}\right) \hat{P}_{3}^{r}+\frac{1}{2} \Omega_{P}(t)\left[\hat{P}_{12}^{r}+\hat{P}_{21}^{r}\right]\right. \\
& \left.+\frac{1}{2} \Omega_{S}(t)\left[\hat{P}_{23}^{r}+\hat{P}_{32}^{r}\right]\right\} \\
= & \frac{\hbar}{2}\left[\begin{array}{ccc}
0 & \Omega_{P}(t) & 0 \\
\Omega_{P}(t) & 2 \Delta_{P} & \Omega_{S}(t) \\
0 & \Omega_{S}(t) & 2\left(\Delta_{P}-\Delta_{S}\right)
\end{array}\right] .
\end{aligned}
$$

Here, $\hat{X}^{r}=e^{i \omega_{P} t \hat{P}_{2}-i\left(\omega_{S}-\omega_{P}\right) t \hat{P}_{3}} \hat{X} e^{-i \omega_{P} t \hat{P}_{2}+i\left(\omega_{S}-\omega_{P}\right) t \hat{P}_{3}} \quad$ (note that $\hat{P}_{k}^{r}=\hat{P}_{k}$ for $\left.k=1,2,3\right)$, and $\Delta_{P}=\omega_{2}-\omega_{1}-\omega_{P}, \Omega_{P}(t)$ $=\mu_{12} \epsilon_{P}(t) / \hbar\left(\Delta_{S}=\omega_{2}-\omega_{3}-\omega_{S}, \Omega_{S}(t)=\mu_{23} \epsilon_{S}(t) / \hbar\right)$ correspond to the detuning and Rabi frequency in the pump (Stokes) transitions. The matrix in Eq. (6) represents $\hat{H}_{s}^{r}$ in terms of the basis of field-free eigenstates (in the RF), $\left\{\left|1^{r}\right\rangle,\left|2^{r}\right\rangle,\left|3^{r}\right\rangle\right\}$, which we will refer to below as the $P$ representation.

From this point on, we will assume that the two-photon resonance condition is satisfied, namely that $\Delta_{P}=\Delta_{S}=\Delta$ (deviations from this condition are known to decrease the efficiency of STIRAP). For this case, we rewrite $\hat{H}_{s}^{r}$ in terms of its instantaneous eigenvalues and eigenstates:

$$
\begin{aligned}
\hat{H}_{s}^{r}(t)= & \frac{\hbar \omega_{+}(t)}{2}\left|a^{+}(t)\right\rangle\left\langle a^{+}(t)\left|+\frac{\hbar \omega_{0}}{2}\right| a^{0}(t)\right\rangle\left\langle a^{0}(t)\right| \\
& +\frac{\hbar \omega_{-}(t)}{2}\left|a^{-}(t)\right\rangle\left\langle a^{-}(t)\right| \\
= & \frac{\hbar}{2}\left[\begin{array}{ccc}
\omega_{+}(t) & 0 & 0 \\
0 & \omega_{0} & 0 \\
0 & 0 & \omega_{-}(t)
\end{array}\right],
\end{aligned}
$$

where, 


$$
\begin{aligned}
\omega_{0}=0, \quad \omega_{ \pm}(t)=\Delta \pm \sqrt{\Delta^{2}+\Omega_{P}^{2}(t)+\Omega_{S}^{2}(t)}, \\
\left|a^{+}\right\rangle=\sin (\theta) \sin (\phi)\left|1^{r}\right\rangle+\cos (\phi)\left|2^{r}\right\rangle \\
+\cos (\theta) \sin (\phi)\left|3^{r}\right\rangle, \\
\left|a^{0}\right\rangle=\cos (\theta)\left|1^{r}\right\rangle-\sin (\theta)\left|3^{r}\right\rangle, \\
\left|a^{-}\right\rangle=\sin (\theta) \cos (\phi)\left|1^{r}\right\rangle-\sin (\phi)\left|2^{r}\right\rangle \\
+\cos (\theta) \cos (\phi)\left|3^{r}\right\rangle,
\end{aligned}
$$

and

$$
\begin{aligned}
& \tan [\theta(t)]=\frac{\Omega_{P}(t)}{\Omega_{S}(t)}, \\
& \tan [\phi(t)]=\frac{\left[\Omega_{S}^{2}(t)+\Omega_{P}^{2}(t)\right]^{1 / 2}}{\left[\Omega_{S}^{2}(t)+\Omega_{P}^{2}(t)+\Delta^{2}\right]^{1 / 2}+\Delta} .
\end{aligned}
$$

From this point on, we will refer to the presentation in terms of $\left\{\left|a_{+}\right\rangle,\left|a_{0}\right\rangle,\left|a_{-}\right\rangle\right\}$as the $A$ representation (it should be noted that both $P$ and $A$ representations are defined in the RF picture).

In STIRAP, population transfer from $\left|1^{r}\right\rangle$ to $\left|3^{r}\right\rangle$, without ever populating $\left|2^{r}\right\rangle$, is achieved through adiabatic passage. The latter is based on the assumption that the Rabi frequencies $\Omega_{P}(t)$ and $\Omega_{S}(t)$ vary slowly over time, such that $|\dot{\theta}| \ll\left|\omega_{ \pm}-\omega_{0}\right|$ (this corresponds to $|\dot{\theta}| \ll \sqrt{\Omega_{P}^{2}+\Omega_{S}^{2}} / 2$ when $\Delta=0$, such that adiabaticity is easier to satisfy when the driving fields are more intense). ${ }^{48}$ STIRAP starts by turning on the Stokes pulse. Thus, as long as the pump pulse is off, $\sin (\theta)=0$ and $\cos (\theta)=1$, such that $\left|a^{+}\right\rangle=\cos (\phi)\left|2^{r}\right\rangle$ $+\sin (\phi)\left|3^{r}\right\rangle,\left|a^{0}\right\rangle=\left|1^{r}\right\rangle$ and $\left|a^{-}\right\rangle=-\sin (\phi)\left|2^{r}\right\rangle+\cos (\phi)\left|3^{r}\right\rangle$. More importantly, the energy gap $\left|\omega_{ \pm}-\omega_{0}\right|$ increases with $\Omega_{S}$. Once the state $\left|1^{r}\right\rangle\left(\equiv\left|a^{0}\right\rangle\right)$ is well separated in energy from the states $\left|a_{ \pm}\right\rangle$, population transfer can start by slowly turning off the Stokes pulse, while simultaneously turning on the pump pulse. This corresponds to slowly changing $\theta$ from 0 to $\pi / 2$, such that $\left|a^{0}\right\rangle$ is adiabatically transformed from $\left|1^{r}\right\rangle$ into $-\left|3^{r}\right\rangle$, without ever populating the intermediate state $\left|2^{r}\right\rangle$. It should be noted that, at least in the case of a truly adiabatic process, the efficiency of STIRAP will not be affected by population transfer from level 2 to other levels. In actual applications, the rate of change of the Rabi frequencies is always finite, and losses due to nonadiabatic population transfer to level 2 are inevitable. However, these losses can be made negligibly small by increasing the intensity of the driving fields (a convenient condition for adiabatic passage is $\Omega_{P, S} t_{d} \gg 1$, where $t_{d}$ is the time delay between the pulses ${ }^{48}$ ). Another important feature of the STIRAP efficiency is its relative insensitivity to the value of the time delay between the Stokes and pump pulses. This is because STIRAP mostly relies on having the Rabi frequencies vary slowly enough, and the pulses overlap to some degree.

\section{STIRAP IN THE PRESENCE OF DECOHERENCE-GENERAL CONSIDERATIONS}

The analysis of STIRAP under decoherence first requires that we define the state of the system in terms of a density operator, $\hat{\rho}_{s}$, instead of the state vector, $|\psi(t)\rangle$. To this end, it is useful to briefly reconsider the description of

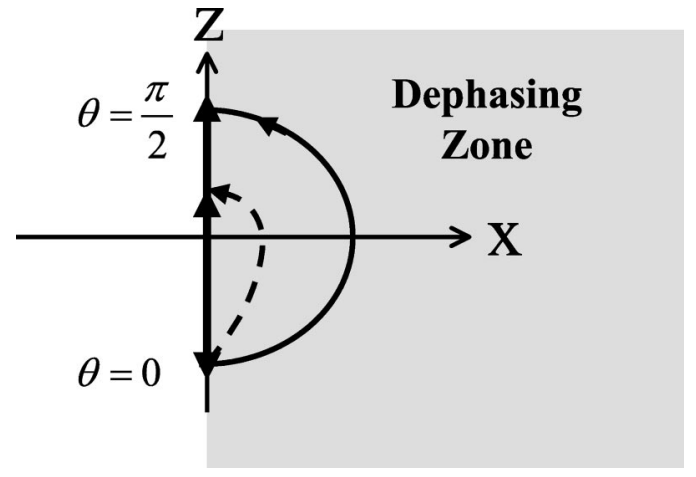

FIG. 2. A description of STIRAP in terms of a rotation of the spinor by $\pi$ in the $x z$ plane. The dynamics is shown in the absence (solid line) and presence (dashed line) of pure dephasing

decoherence-free STIRAP within the framework of the density operator formalism. It is important to note that, at least ideally, the state of a system undergoing STIRAP is restricted to the sub-space spanned by the two states $\left|1^{r}\right\rangle$ and $\left|3^{r}\right\rangle$. This makes it possible to describe STIRAP in terms of a fictitious spin-1/2 system. Thus, the corresponding density operator, can be given in terms of the corresponding "spin" operators:

$$
\hat{\rho}_{s}=\frac{1}{2} \hat{I}+\frac{1}{\hbar}\left[P_{x}^{r} \hat{P}_{x}^{r}+P_{y}^{r} \hat{P}_{y}^{r}+P_{z}^{r} \hat{P}_{z}^{r}\right],
$$

where $\hat{P}_{x}^{r}=\hbar\left(\hat{P}_{13}^{r}+\hat{P}_{31}^{r}\right) / 2, \hat{P}_{y}^{r}=i \hbar\left(\hat{P}_{13}^{r}-\hat{P}_{31}^{r}\right) / 2, \hat{P}_{z}^{r}=\hbar\left(\hat{P}_{3}^{r}\right.$ $\left.-\hat{P}_{1}^{r}\right) / 2$, and $X=\langle\hat{X}\rangle=\operatorname{Tr}\left(\hat{\rho}_{s} \hat{X}\right)$. The state of the system can, therefore, be given in terms of a spinor (or Blochvector), i.e., a vector in a fictitious $(x, y, z)$ Cartesian coordinate system, whose components are $P_{x}^{r}, P_{y}^{r}$ and $P_{z}^{r}$. Within this picture, decoherence-free STIRAP amounts to a rotation of the spinor by $\pi$ in the $(x, z)$ plane, from being aligned along the negative $z$ axis, to being aligned along the positive $z$ axis (cf. Fig. 2). It is important to note that the system remains in a pure state throughout this process. This implies that the corresponding dynamics involves the rotation of the spinor, without changing its amplitude $\left(\operatorname{Tr}\left[\hat{\rho}_{s}^{2}(t)\right]\right.$ $\left.=\operatorname{Tr}\left[\hat{\rho}_{s}(t)\right]=1\right)$. Such a unitary transformation is only possible if decoherence is negligible.

The main goal of this paper is to examine what happens to STIRAP when decoherence is not negligible on the time scale of the experiment. To this end, it is useful to distinguish between two fundamentally different types of relaxation, namely population relaxation, and dephasing (phase relaxation). Population relaxation involves the diagonal elements of $\hat{\rho}_{s}$ (in the $P$ representation), and leads to the relaxation of the $P_{z}^{r}$ component of the spinor, to its value at thermal equilibrium. Dephasing involves the off-diagonal elements of $\hat{\rho}_{s}$, and leads to the relaxation of the $P_{x}^{r}$ and $P_{y}^{r}$ components of the spinor to zero. Dephasing can be further decomposed into two components, one that originates from population relaxation, and another that does not. The latter is referred to as pure dephasing.

STIRAP would be rather ineffective if the life-time of population relaxation from levels 1 and 3 is comparable to, or shorter than, the experimental time scale (conveniently 
defined by the time delay between the Stokes and pump pulses). In such a case, any deviation from the Boltzmann distribution brought about by STIRAP, would be quickly offset by population relaxation. In addition, population relaxation is known to be strongly dependent on the spectral density of the solvent, and controlling it would require very intense fields, with a Rabi frequencies comparable to the transition frequencies. ${ }^{51,52}$ It is therefore a rather fortunate coincidence that population relaxation is often found to be significantly slower than pure dephasing. ${ }^{30}$ This is particularly true in the case of a diatomic solute embedded in an atomic liquid, where population relaxation involves relatively inefficient vibration-rotation and vibration-translation energy transfer. For example, Everitt and Skinner have recently calculated the life time of the first excited vibrational state of oxygen in liquid Argon from MD simulations, and reported a value as long as $0.04 \mathrm{~s}^{-1}$ at $85.8 \mathrm{~K} .{ }^{53}$ For these reasons, we will assume that the population relaxation lifetime is long compared to the experimental time scale, and restrict ourselves to the study of STIRAP in the presence of only pure dephasing.

The influence of pure dephasing on STIRAP can be qualitatively understood in terms of the above mentioned spinor-based picture. As long as the spinor is aligned along the $z$ axis, as in the initial and final states, it will not be influenced by pure dephasing. However, rotating the spinor by $\pi$ in the $x z$ plane immediately exposes its $x$ component to pure dephasing, which will diminish its overall amplitude (cf. Fig. 2). This will obviously result in less than complete population transfer (in the extreme limit of very fast pure dephasing, the populations in the $\left|1^{r}\right\rangle$ and $\left|3^{r}\right\rangle$ states would be equal at the end of the experiment). It is therefore clear that pure dephasing will reduce the efficiency of population transfer via STIRAP, unless it can somehow be effectively slowed down.

At this point, it is instructive to look more closely at the type of solute-solvent interactions that give rise to pure dephasing. To this end, we introduce the following general system-bath Hamiltonian:

$$
\hat{H}=\hat{H}_{s}^{0}+\hat{W}(t)+\hat{H}_{b}+\hat{H}_{b s},
$$

where $\hat{H}_{s}^{0}$ and $\hat{W}(t)$ are as in Eqs. (1) and (2), respectively, $\hat{H}_{b}$ is the bath Hamiltonian, which corresponds to the other intermolecular and intramolecular DOF and will remain unspecified for the time being, and $\hat{H}_{b s}$ is the system-bath coupling term

$$
\hat{H}_{b s}=\hat{\Gamma}_{S} \otimes \frac{1}{2}\left(\hat{P}_{2}-\hat{P}_{3}\right)+\hat{\Gamma}_{P} \otimes \frac{1}{2}\left(\hat{P}_{2}-\hat{P}_{1}\right) .
$$

Here $\hat{\Gamma}_{S}$ and $\hat{\Gamma}_{P}$ are bath operators, which will not be explicitly specified for the time being.

It should be noted that $\hat{H}_{b s}$ in Eq. (13) only includes diagonal system-bath coupling, i.e., coupling terms that only involve the operators $\hat{P}_{1}, \hat{P}_{2}$, and $\hat{P}_{3}$. Such terms lead to pure dephasing, but not to population relaxation (population relaxation originates from off-diagonal coupling terms that involve the operators $\hat{P}_{i j}$, where $i \neq j$ ). We also note that $\hat{H}_{b s}$ can be rewritten in the following way:

$$
\hat{H}_{b s}=-\frac{1}{2} \hat{\Gamma}_{P} \otimes \hat{P}_{1}+\frac{1}{2}\left(\hat{\Gamma}_{S}+\hat{\Gamma}_{P}\right) \otimes \hat{P}_{2}-\frac{1}{2} \hat{\Gamma}_{S} \otimes \hat{P}_{3} .
$$

Thus, $-\hat{\Gamma}_{P} / 2,\left(\hat{\Gamma}_{S}+\hat{\Gamma}_{P}\right) / 2$ and $-\hat{\Gamma}_{S} / 2$ represent the fluctuations of the individual energy levels of the states $|1\rangle,|2\rangle$, and $|3\rangle$, respectively. The explicit form of $\hat{\Gamma}_{S}$ and $\hat{\Gamma}_{P}$, as well as the relationship between them, depends on the specific system. Below, we will consider the following three general scenarios:

(1) correlated coupling, where $\Gamma_{S}=\Gamma_{P}$;

(2) anti-correlated coupling, where $\Gamma_{S}=-\Gamma_{P}$;

(3) uncorrelated coupling, where $\operatorname{Tr}\left(e^{-\beta \hat{H}_{b}} \hat{\Gamma}_{S} \hat{\Gamma}_{P}\right)=0$.

Coupling the three-level STIRAP system to a bath turns it into an open quantum system. Its dynamics is therefore not Hamiltonian, and cannot be described by the Schrödinger equation. The (reduced) dynamics of such an open quantum system is dictated by an equation of motion ${ }^{54}$ of the following general form:

$$
\begin{aligned}
\frac{d}{d t} \hat{\rho}_{s}(t)= & -\frac{i}{\hbar} \mathcal{L}_{s}^{0} \hat{\rho}_{s}(t)-\frac{i}{\hbar} \mathcal{L}_{W}(t) \hat{\rho}_{s}(t) \\
& +\int_{0}^{\infty} d \tau \mathcal{K}(t, t-\tau) \hat{\rho}_{s}(t-\tau),
\end{aligned}
$$

where, $\mathcal{L}_{s}^{0} \hat{\rho}(t)=\left[\hat{H}_{s}^{0}, \hat{\rho}(t)\right]$ and $\mathcal{L}_{W}(t) \hat{\rho}(t)=[\hat{W}(t), \hat{\rho}(t)]$ represent the dephasing-free Hamiltonian dynamics, while $\int_{0}^{\infty} d \tau \mathcal{K}(t, t-\tau) \hat{\rho}(t-\tau)$ represents the non-Hamiltonian, and non-Markovian, bath-induced dynamics. Unfortunately, Eq. (15) is of little practical use as such, due to the complexity of $\mathcal{K}(t, t-\tau) .{ }^{54,55}$ In this paper, we resort to the commonly used weak system-bath coupling limit of $\mathcal{K}(t, t-\tau)$ as a way of simplifying the description. We also assume that the correlation-time of the bath fluctuations, $\tau_{c}$, is considerably shorter than the pure-dephasing lifetime. As is well known, these two assumptions make it possible to describe the reduced dynamics in terms of a Markovian QME of the form: ${ }^{54,55}$

$$
\frac{d}{d t} \hat{\rho}(t)=-\frac{i}{\hbar} \mathcal{L}_{s}^{0} \hat{\rho}(t)-\frac{i}{\hbar} \mathcal{L}_{W}(t) \hat{\rho}(t)+\mathcal{L}_{D} \hat{\rho}(t) .
$$

However, the specific form of the dissipative super-operator $\mathcal{L}_{D}$ still depends on how we treat the field-matter interaction term. The most popular approach is based on the, often implicit, assumption that the form of $\mathcal{L}_{D}$ is the same as it would have been in the absence of the driving fields. However, this treatment is strictly valid only in the limit of weak fieldmatter interaction. In the cases which are of interest for us in this paper, this translates into assuming that the Rabi frequency of the driving field is small relative to $\tau_{c}^{-1}$ (cf. Sec. IV and the Appendix for a more detailed discussion of this point). A more general treatment, which avoids any assumption regarding the field-matter interaction and is therefore valid for driving fields of arbitrary intensity, is nevertheless possible in the case of STIRAP. It leads to a modified $\mathcal{L}_{D}$, which is explicitly dependent on the driving fields (cf. Sec. V for a more detailed discussion of this point), thereby opening the door for using the field for actively controlling, and pos- 
TABLE I. The pure-dephasing rate constants in the correlated $\left[C_{S}(\tau)=C_{P}(\tau)=C_{P S}(\tau)=C(\tau)\right]$, anticorrelated $\left[C_{S}(\tau)=C_{P}(\tau)=-C_{P S}(\tau)=C(\tau)\right]$, and uncorrelated $\left[C_{P S}(\tau)=0\right]$ coupling schemes.

\begin{tabular}{lccc}
\hline \hline & $\hbar^{2} \kappa_{12}$ & $\hbar^{2} \kappa_{23}$ & $\hbar^{2} \kappa_{13}$ \\
\hline Correlated & $9 \widetilde{C}(0) / 8$ & $9 \widetilde{C}(0) / 8$ & 0 \\
Anti-correlated & $\widetilde{C}(0) / 8$ & $\widetilde{C}(0) / 8$ & $\widetilde{C}(0) / 2$ \\
Uncorrelated & $\widetilde{C}_{P}(0) / 2+\widetilde{C}_{S}(0) / 8$ & $\widetilde{C}_{P}(0) / 8+\widetilde{C}_{S}(0) / 2$ & $\widetilde{C}_{P}(0) / 8+\widetilde{C}_{S}(0) / 8$ \\
\hline \hline
\end{tabular}

sibly suppressing, pure dephasing. The impact of pure dephasing on STIRAP in these two cases is discussed in detail in Secs. IV and V.

\section{STIRAP UNDER FIELD-INDEPENDENT PURE DEPHASING (WEAK DRIVING FIELDS)}

In this section, we present a more quantitative analysis of the influence of pure dephasing over STIRAP, in the case of weak field-matter coupling (in the sense that $\Omega \tau_{c} \ll 1$ ). As shown in the Appendix, the dissipative super-operator $\mathcal{L}_{D}$ becomes field-independent in this case, and assumes the exact same form as it would have had in the absence of the driving field. In the case of STIRAP, it is convenient to perform the derivation of the QME in terms of the RF picture. To this end, we define the density operator of the overall system (system + bath) in the RF picture

$$
\hat{\rho}^{r}(t)=e^{i \omega_{P} t \hat{P}_{2}-i\left(\omega_{S}-\omega_{P}\right) t \hat{P}_{3}} \hat{\rho}(t) e^{-i \omega_{P} t \hat{P}_{2}+i\left(\omega_{S}-\omega_{P}\right) t \hat{P}_{3}},
$$

where $\hat{\rho}(t)$ is the density operator in the original Schrödinger picture. The dynamics of $\hat{\rho}^{r}(t)$ is dictated by the Liouville equation

$$
\frac{d}{d t} \hat{\rho}^{r}(t)=-\frac{i}{\hbar}\left[\hat{H}^{r}, \hat{\rho}^{r}(t)\right],
$$

where

$$
\hat{H}^{r}=\hat{H}_{s}^{r}+\hat{H}_{b}^{r}+\hat{H}_{b s}^{r},
$$

$\hat{H}_{s}^{r}$ is as in Eq. (6), $\hat{H}_{b}^{r}=\hat{H}_{b}$, and

$$
\hat{H}_{b s}^{r}=\hat{\Gamma}_{S} \otimes \frac{1}{2}\left(\hat{P}_{2}^{r}-\hat{P}_{3}^{r}\right)+\hat{\Gamma}_{P} \otimes \frac{1}{2}\left(\hat{P}_{2}^{r}-\hat{P}_{1}^{r}\right) .
$$

Using the RF Hamiltonian in Eq. (19) as the starting point for the derivation, and assuming weak field-matter interaction, such that the dissipation term is the same as it would have been in the absence of the driving term [i.e., when $\Omega_{P}, \Omega_{S}=0$ in Eq. (6)], then leads to a QME of the following form (cf. the Appendix):

$$
\frac{d}{d t} \hat{\rho}_{s}^{r}=-\frac{i}{\hbar} \mathcal{L}_{s}^{r} \hat{\rho}^{r}+\mathcal{L}_{D}^{0} \hat{\rho}_{s}^{r},
$$

where $\mathcal{L}_{s}^{r} \hat{\rho}_{s}^{r}=\left[\hat{H}_{s}^{r}, \hat{\rho}_{s}^{r}\right]$ is the Hamiltonian term, and

$$
\begin{aligned}
\mathcal{L}_{D}^{0} \hat{\rho}_{s}^{r}= & -\frac{1}{2 \hbar^{2}}\left\{\widetilde{C}_{P}(0)\left[\left(\hat{P}_{2}^{r}-\hat{P}_{1}^{r}\right) / 2,\left[\left(\hat{P}_{2}^{r}-\hat{P}_{1}^{r}\right) / 2, \hat{\rho}_{s}^{r}\right]\right]\right. \\
& +\widetilde{C}_{S}(0)\left[\left(\hat{P}_{2}^{r}-\hat{P}_{3}^{r}\right) / 2,\left[\left(\hat{P}_{2}^{r}-\hat{P}_{3}^{r}\right) / 2, \hat{\rho}_{s}^{r}\right]\right] \\
& +\widetilde{C}_{P S}(0)\left(\left[\left(\hat{P}_{2}^{r}-\hat{P}_{1}^{r}\right) / 2,\left[\left(\hat{P}_{2}^{r}-\hat{P}_{3}^{r}\right) / 2, \hat{\rho}_{s}^{r}\right]\right]\right. \\
& \left.\left.+\left[\left(\hat{P}_{2}^{r}-\hat{P}_{3}^{r}\right) / 2,\left[\left(\hat{P}_{2}^{r}-\hat{P}_{1}^{r}\right) / 2, \hat{\rho}_{s}^{r}\right]\right]\right)\right\} .
\end{aligned}
$$

Here

$$
\widetilde{C}_{k l}(\omega)=\int_{-\infty}^{\infty} d \tau C_{k l}(\tau) e^{i \omega \tau},
$$

is the Fourier transform of the bath correlation function

$$
C_{k l}(\tau)=\operatorname{Tr}\left(\hat{\rho}_{b}^{\mathrm{eq}} e^{i \hat{H}_{b} \tau / \hbar} \hat{\Gamma}_{k} e^{-i \hat{H}_{b} \tau / \hbar} \hat{\Gamma}_{l}\right)
$$

where $k, l=P, S, \widetilde{C}_{k k}(\omega)=\widetilde{C}_{k}(\omega)$ and $\hat{\rho}_{b}^{\mathrm{eq}}$ is as in Eq. (A5). It should be emphasized that the Hamiltonian term in Eq. (21), $-i \mathcal{L}_{s}^{r} \hat{\rho}^{r} / \hbar$, includes the driving terms to all orders, whereas the dissipative term, $\mathcal{L}_{D}^{0} \hat{\rho}^{r}$, is the same as it would have been in its absence.

Equation (21) translates into the following set of coupled equations for the populations and coherences in the $P$ representation:

$$
\begin{aligned}
& \dot{P}_{1}^{r}=\Omega_{P} \operatorname{Im} P_{12}^{r}, \\
& \dot{P}_{2}^{r}=-\Omega_{P} \operatorname{Im} P_{12}^{r}+\Omega_{S} \operatorname{Im} P_{23}^{r}, \\
& \dot{P}_{3}^{r}=-\Omega_{S} \operatorname{Im} P_{23}^{r}, \\
& \dot{P}_{12}^{r}=i \Omega_{P}\left(P_{2}^{r}-P_{1}^{r}\right) / 2-i \Omega_{S} P_{13}^{r} / 2-\left(i \Delta+\kappa_{12}\right) P_{12}^{r}, \\
& \dot{P}_{23}^{r}=-i \Omega_{S}\left(P_{2}^{r}-P_{3}^{r}\right) / 2+i \Omega_{P} P_{13}^{r} / 2-\left(i \Delta+\kappa_{23}\right) P_{23}^{r}, \\
& \dot{P}_{13}^{r}=i \Omega_{P} P_{23}^{r} / 2-i \Omega_{S} P_{12}^{r} / 2-\kappa_{13} P_{13}^{r} .
\end{aligned}
$$

Equations (25) clearly do not involve bath-induced population relaxation. However, the coupling to the bath does give rise to dephasing, and the pure dephasing rate constants are given by

$$
\begin{aligned}
\kappa_{12} & =\frac{1}{2 \hbar^{2}}\left(\widetilde{C}_{P}(0)+\frac{1}{4} \widetilde{C}_{S}(0)+\widetilde{C}_{P S}(0)\right), \\
\kappa_{23} & =\frac{1}{2 \hbar^{2}}\left(\frac{1}{4} \widetilde{C}_{P}(0)+\widetilde{C}_{S}(0)+\widetilde{C}_{P S}(0)\right), \\
\kappa_{13} & =\frac{1}{2 \hbar^{2}}\left(\frac{1}{4} \widetilde{C}_{P}(0)+\frac{1}{4} \widetilde{C}_{S}(0)-\frac{1}{2} \widetilde{C}_{P S}(0)\right) .
\end{aligned}
$$


In order to gain further insight into the influence of pure dephasing on STIRAP, it is useful to refer to the special cases of correlated $\left[C_{S}(\tau)=C_{P}(\tau)=C_{P S}(\tau)=C(\tau)\right]$, anticorrelated $\left[C_{S}(\tau)=C_{P}(\tau)=-C_{P S}(\tau)=C(\tau)\right]$, and uncorrelated $\left[C_{P S}(\tau)=0\right]$ coupling schemes. The corresponding expressions for the pure dephasing rate constants are given in Table I. It should be noted that $\kappa_{13}$ is minimized (and actually vanishes), while $\kappa_{12}$ and $\kappa_{23}$ are maximized in the correlated case. The opposite happens in the anti-correlated case, where $\kappa_{13}$ is maximized, while $\kappa_{12}$ and $\kappa_{23}$ are minimized. Thus, one expects that STIRAP, which relies heavily on maintaining $P_{13}$ during adiabatic passage, will be protected from dephasing in the case of correlated dephasing, and destroyed by it in the case of anti-correlated dephasing. The case of uncorrelated dephasing corresponds to an intermediate situation, where dephasing is destructive, but to a lesser extent than in the anti-correlated case.

We have also performed numerical simulations of the STIRAP process in a system whose dynamics is dictated by Eqs. (25). The pump and Stokes pulses were assumed to have the same shape, such that:

$$
\Omega_{S}(t)=\Omega_{P}\left(t+t_{d}\right)=\Omega_{0} \sin ^{4}\left(\pi t / t_{0}\right) \text { for } 0 \leqslant t \leqslant t_{0},
$$

where $t_{d}$ is the delay between the peaks of the Stokes and pump pulses, $t_{0}$ is the pulse width and $\Omega_{0}$ is the maximal Rabi frequency. The results will be presented below in terms of dimensionless variables which are all scaled relative to $t_{0}$. The following values of the parameters have been used in the actual simulations: $\Omega_{0} t_{0}=240$ and $\Delta=0$ (one-photon resonance). The time origin has been set at the start of the Stokes pulse. It should be noted that $\Omega_{0} t_{0} \gg 1$, and therefore satisfies the condition for efficient adiabatic passage. ${ }^{48}$ The dephasing parameters were chosen such that $\widetilde{C}(0) t_{0}=96.0 \hbar^{2}$. Finally, we have also added population relaxation from level 2 into levels other than 1 and 3, such that the equation of motion of $P_{2}^{r}$ is given by

$$
\dot{P}_{2}^{r}=-\Omega_{P} \operatorname{Im} P_{12}^{r}+\Omega_{S} \operatorname{Im} P_{23}^{r}-T_{1}^{-1} P_{2}^{r} .
$$

The value of $\left(T_{1} / t_{0}\right)^{-1}=30.0$ has been used in the actual simulations. It should be noted that the values of $\widetilde{C}(0) / \hbar^{2}$ and $T_{1}$ were chosen such that dephasing and population relaxation are significant on the experimental time scale, which is set by $t_{0}$. The propagation of Eqs. (25) has been performed with the standard fourth-order Runge-Kutta method. ${ }^{56}$

In Fig. 3, we show the simulated final value of $P_{3}^{r}$, at the end of the pump pulse $\left(t=t_{0}+t_{d}\right)$, as a function of the time delay between the Stokes and pump pulses, $t_{d}$. The results are shown in the absence of dephasing (0), and for the cases of correlated (C), uncorrelated (U), and anti-correlated (A) dephasing. STIRAP is seen to be very efficient in the absence of dephasing, with a transfer efficiency of nearly $100 \%$, and a very small loss due to nonadiabatic transitions to level 2 . As is well known, the dephasing-free performance is rather insensitive to the actual value of the time delay, as indicated by the wide range of $t_{d}$ values that give rise to nearly $100 \%$ transfer efficiency.

In Fig. 4 we show the time evolution of $P_{1}^{r}, P_{2}^{r}, P_{3}^{r}$,

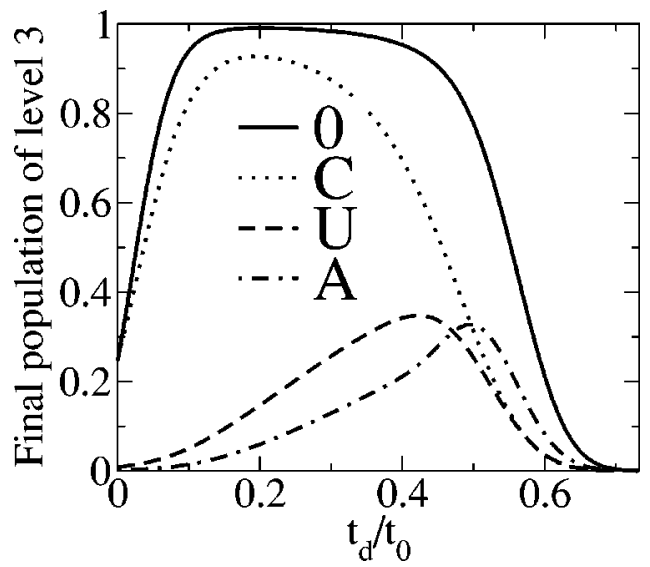

FIG. 3. The final value of $P_{3}^{r}$ as a function of the time delay between the Stokes and pump pulses. The results are shown in the absence of dephasing $(0)$, and for the cases of correlated (C), uncorrelated (U), and anti-correlated (A) dephasing. The parameters used in the simulation are given in the text.

$\operatorname{Im} P_{12}^{r}, \operatorname{Im} P_{23}^{r}$, and $\operatorname{Re} P_{13}^{r}$ throughout the dephasing-free STIRAP process, for a specific time delay $\left(t_{d} / t_{0}=1 / 3\right)$. It should be noted that the real parts of $P_{12}^{r}$ and $P_{23}^{r}$ vanish when $\Delta=0$. The population transfer is seen to be accompanied by a buildup followed by a decrease of $P_{13}^{r}$, which reflects the rotation of the spinor in the $x z$ plane (cf. Fig. 2). Deviations from adiabaticity are observed in the form of small buildups of $P_{2}^{r}, P_{12}^{r}$, and $P_{23}^{r}$ during the period of overlap between the Stokes and pump pulses. It is important to note that the population is restricted to level 1 until the pump pulse is turned on. Thus, $P_{12}^{r}$ is first to emerge, followed by the creation of population in level $2, P_{2}^{r}$, which in turn gives rise to $P_{23}^{r}$. It is also important to note that the net rate of populating level 2 is proportional to the difference between the (Rabi-frequency-weighted) imaginary parts of $P_{12}^{r}$ and $P_{23}^{r}$ [cf. Eqs. (25)]. This difference is attributed to the delay between the buildups of $P_{12}^{r}$ and $P_{23}^{r}$, which otherwise have the same shape.

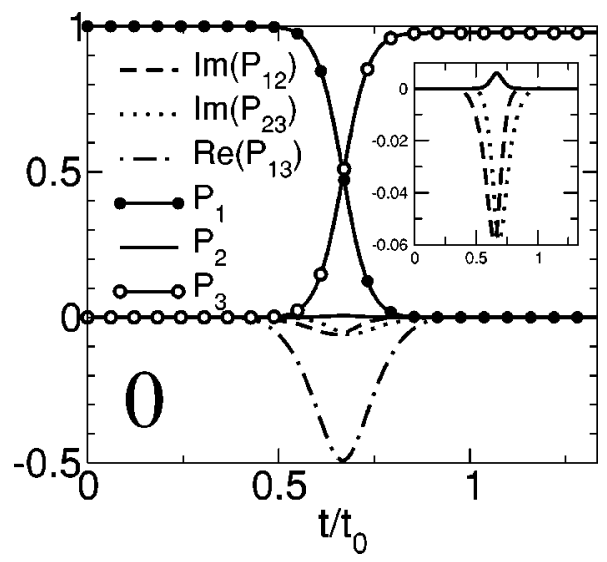

FIG. 4. The time evolution of $P_{1}^{r}, P_{2}^{r}, P_{3}^{r}, \operatorname{Im} P_{12}^{r}, \operatorname{Im} P_{23}^{r}$, and $\operatorname{Re} P_{13}^{r}$ throughout the dephasing-free STIRAP process, for $t_{d} / t_{0}=1 / 3$. The Stokes (pump) pulse is turned on at $t / t_{0}=0\left(t / t_{0}=1 / 3\right)$, peaks at $t / t_{0}=1 / 2\left(t / t_{0}\right.$ $=5 / 6)$ and is turned off at $t / t_{0}=1.0\left(t / t_{0}=4 / 3\right)$. Note that $P_{13}^{r}$ is purely real, while $P_{12}^{r}$ and $P_{23}^{r}$ are purely imaginary throughout the dynamics. The inset shows a close-up of $P_{2}^{r}, \operatorname{Im} P_{12}^{r}$, and $\operatorname{Im} P_{23}^{r}$. 


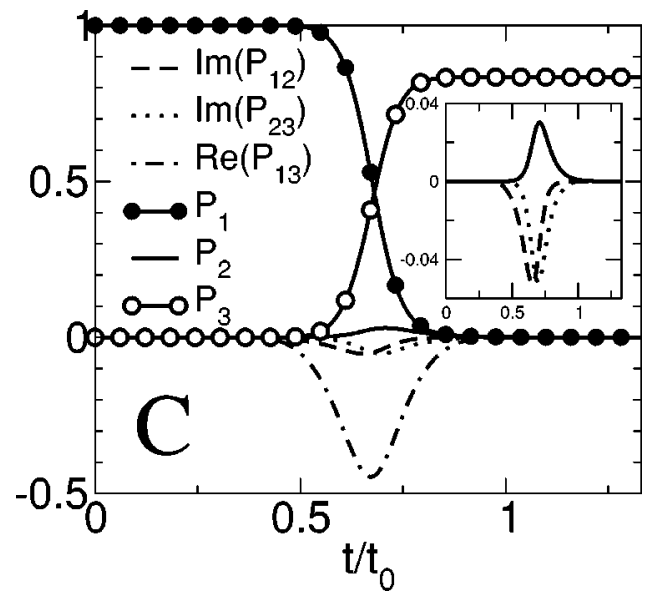

FIG. 5. Same as Fig. 4 for STIRAP under correlated dephasing.

The STIRAP efficiency under correlated dephasing $(\mathrm{C}$ in Fig. 3) is seen to be relatively high ( $\sim 93 \%$ at the peak), but visibly lower than in the dephasing-free case. This is somewhat surprising in light of the fact that $\kappa_{13}=0$ in this case (cf. Table I). It should also be emphasized that neither the dissipative term nor the Hamiltonian term in the QME can independently lead to additional population transfer to level 2 (the former because it can only account for pure dephasing, and the latter because of adiabaticity). Thus, the reduction in efficiency in the case of correlated dephasing must be the result of enhanced nonadiabaticity, which is due to dephasing. Further insight into this process can be gained from Fig. 5 which shows the time evolution of $P_{1}^{r}, P_{2}^{r}, P_{3}^{r}, \operatorname{Im} P_{12}^{r}$, $\operatorname{Im} P_{23}^{r}$, and $\operatorname{Re} P_{13}^{r}$ under correlated dephasing, for a specific time delay $\left(t_{d} / t_{0}=1 / 3\right)$. It should be noted that the overall buildup of $P_{12}^{r}$ and $P_{23}^{r}$ is smaller in comparison to the dephasing-free case, which is attributed to the fact that $\kappa_{12}$ and $\kappa_{23}$ are maximized in the case of correlated dephasing. However, the dephasing appears to also break the symmetry in the shapes of the $P_{12}^{r}$ and $P_{23}^{r}$ buildups, such that the difference between them is larger than that in the dephasingfree case, which results in a somewhat larger rate of population transfer into level 2.

The STIRAP efficiency drops dramatically and becomes much more sensitive to the value of $t_{d}$ under uncorrelated dephasing (U in Fig. 3). Figure 6 shows the time evolution of $P_{1}^{r}, P_{2}^{r}, P_{3}^{r}, \operatorname{Im} P_{12}^{r}, \operatorname{Im} P_{23}^{r}$, and $\operatorname{Re} P_{13}^{r}$ in this case, for a specific time delay $\left(t_{d} / t_{0}=1 / 3\right.$ ). In this case, $\kappa_{13} \neq 0$ (cf. Table I), and the dephasing of $P_{13}^{r}$ is clearly manifested by its diminished buildup (cf. Fig. 2). Furthermore, the dephasing is found to break the symmetry in the shapes of the $P_{12}^{r}$ and $P_{23}^{r}$ buildups in a much more pronounced manner, thereby leading to enhanced nonadiabaticity that add further losses. It is interesting to note that the buildup of $P_{23}^{r}$ is significantly smaller than that of $P_{12}^{r}$, despite the fact that they are both subject to the same dephasing rate constants $\left(\kappa_{12}=\kappa_{23}\right.$, cf. Table I). The reason for this can be traced back to the fact that the Stokes and pump fields, which are the driving forces behind the creation of $P_{23}^{r}$ and $P_{12}^{r}$, respectively, must work against pure dephasing, which tends to destroy them. Because the Stokes field decreases and the pump pulse

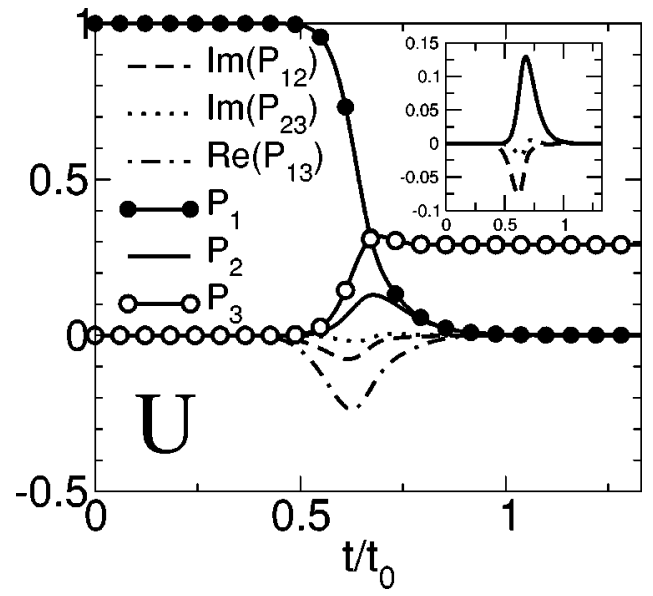

FIG. 6. Same as Fig. 4 for STIRAP under uncorrelated dephasing.

increases during the overlap time, the "sustaining power" of $P_{23}^{r}$ is lower that that of $P_{12}^{r}$. Hence, the smaller buildup of the former.

It is also interesting to note that the maximal efficiency in the case of uncorrelated dephasing ( $\mathrm{U}$ in Fig. 3), corresponds to a relatively long delay from the range of delays that give close to $100 \%$ efficiency in the dephasing-free case (0 in Fig. 3). This observation is consistent with the fact that a longer delay corresponds to a shorter period of overlap between the pulses, which in turn implies shorter exposure times to dephasing. It should be remembered that some degree of overlap is essential if STIRAP is to take place, and that this maximum represents a compromise between adiabaticity, which benefits from longer overlap times, and dephasing, whose destructive effect also increases with the overlap time.

Finally, the STIRAP efficiency is seen to drop in value, as well as become even more sensitive to the value of $t_{d}$ and peak at an even longer delay, under anti-correlated dephasing (A in Fig. 3). Figure 7 shows the time evolution of $P_{1}^{r}, P_{2}^{r}$, $P_{3}^{r}, \operatorname{Im} P_{12}^{r}, \operatorname{Im} P_{23}^{r}$, and $\operatorname{Re} P_{13}^{r}$ in this case, for a specific time delay $\left(t_{d} / t_{0}=1 / 3\right.$ ). In this case, $\kappa_{13}$ is maximized (cf. Table I), which is clearly manifested by the minimization of

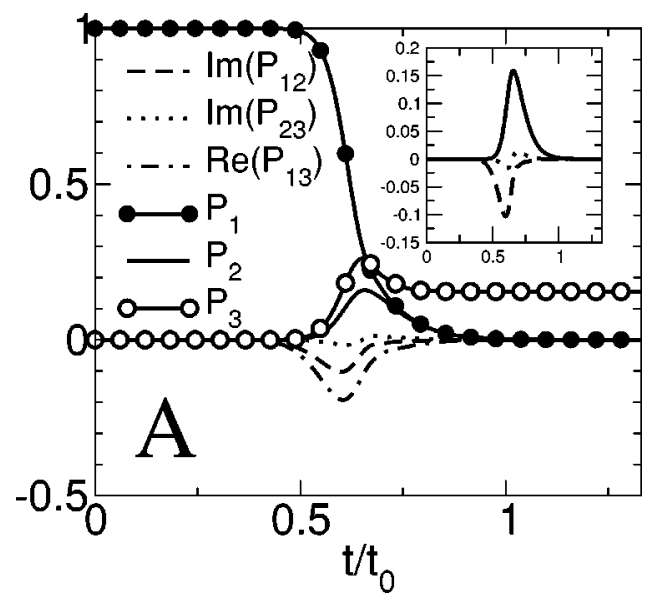

FIG. 7. Same as Fig. 4 for STIRAP under anti-correlated dephasing. 
TABLE II. The relaxation rate constants for correlated dephasing $\left[C_{S}(\tau)=C_{P}(\tau)=C_{P S}(\tau)=C(\tau)\right]$. The entry at the $i j$ line and $k l$ column correspond to $\hbar^{2} \gamma_{i j k l}$. The upper (lower) signs in the $(k l, l k)$ column entries correspond to $k l(l k) . \nu_{ \pm}=\omega_{ \pm} / 2 . s_{\phi}, c_{\phi}, s_{\theta}$, and $c_{\theta}$ correspond to $\sin \phi, \cos \phi, \sin \theta$, and $\cos \theta$, respectively. Note that $\gamma_{i j k l}=\gamma_{j i k l}$. Entries that are not explicitly shown are equal to zero.

\begin{tabular}{lccc}
\hline \hline & ++ & -- &,+--+ \\
\hline++ & $\frac{1}{2}\left(1-3 c_{\phi}^{2}\right)^{2} \widetilde{C}(0)$ & $\frac{1}{2}\left(1-3 c_{\phi}^{2}\right)\left(1-3 s_{\phi}^{2}\right) \widetilde{C}(0)$ & $\frac{3}{4} c_{\phi} s_{\phi}\left(3 c_{\phi}^{2}-1\right) \widetilde{C}\left(\mp \nu_{+} \mp \nu_{-}\right)$ \\
-- & $\frac{1}{2}\left(1-3 c_{\phi}^{2}\right)\left(1-3 s_{\phi}^{2}\right) \widetilde{C}(0)$ & $\frac{1}{2}\left(1-3 s_{\phi}^{2}\right)^{2} \widetilde{C}(0)$ & $\frac{3}{4} c_{\phi} s_{\phi}\left(3 s_{\phi}^{2}-1\right) \widetilde{C}\left(\mp \nu_{+} \mp \nu_{-}\right)$ \\
+- & $\frac{3}{4} c_{\phi} s_{\phi}\left(3 c_{\phi}^{2}-1\right) \widetilde{C}(0)$ & $\frac{3}{4} c_{\phi} s_{\phi}\left(3 s_{\phi}^{2}-1\right) \widetilde{C}(0)$ & $\frac{9}{8} c_{\phi}^{2} s_{\phi}^{2} \widetilde{C}\left(\mp \nu_{+} \mp \nu_{-}\right)$ \\
-+ & & & \\
\hline \hline
\end{tabular}

the buildup in $P_{13}^{r}$. The asymmetry in the buildups of $P_{12}^{r}$ and $P_{23}^{r}$ is also enhanced in this case, and leads to an increased loss of population through level 2 .

\section{STIRAP UNDER FIELD-DEPENDENT PURE DEPHASING (INTENSE DRIVING FIELDS)}

The validity of the QME used to describe STIRAP in Sec. IV is limited to weak driving fields (in the sense that $\left.\Omega \tau_{c} \ll 1\right)$. However, the fact that the Rabi period is required to be longer than the STIRAP delay time, in order to satisfy adiabaticity, implies that a weak field treatment of the dissipative part may be inconsistent. To this end, we extend the treatment in this section, to driving fields of arbitrary intensity. As in the previous section, we start with the Liouville equation for the density operator of the overall system in the RF, Eq. (18). However, we restrict the perturbative treatment to the system-bath interaction term, $\hat{H}_{b s}$, and refrain from applying it to the field-matter interaction term (cf. the Appendix).

It should be noted that the field-matter interaction term is stationary in the RF, except for the time dependence of the pulse envelopes, $\Omega_{S}(t)$ and $\Omega_{P}(t)$. Adiabaticity dictates that $\Omega_{S}(t)$ and $\Omega_{P}(t)$ change on the time scale of the pulse length, that was assumed to be longer than the dephasing lifetime, which is in turn longer than the bath correlation time, $\tau_{c}$ (Markovity). Thus, $\Omega_{S}(t)$ and $\Omega_{P}(t)$ can be assumed to be constant over a period of the bath correlation time, such that the dissipative term has the same form as it would have had in the case of a truly stationary system that corresponds to the instantaneous Hamiltonian, $\hat{H}_{s}^{r}(t)$. As a result, the energy levels and eigen-projectors in the QME correspond to the A representation and are, therefore, explicitly time-dependent [cf. Eq. (A16) in the Appendix].

In light of the above, it is convenient to perform the actual derivation of the QME in terms of the $A$ representation. To this end, we introduce the instantaneous eigenprojectors of $\hat{H}_{s}^{r}(t)$ :

$$
\hat{A}_{k l}(t)=\left|a^{k}(t)\right\rangle\left\langle a^{l}(t)\right| \text { where } k, l=+, 0,- \text {. }
$$

Obviously, $\quad \hat{H}_{s}^{r}(t)=(\hbar / 2)\left[\omega_{+}(t) \hat{A}_{++}(t)+\omega_{0}(t) \hat{A}_{00}(t)\right.$ $\left.+\omega_{-}(t) \hat{A}_{--}(t)\right]$. The system-bath coupling term $\hat{H}_{b s}[\mathrm{cf}$. Eq. (13)] assumes the following form in the $A$ representation:

$$
\begin{aligned}
\hat{H}_{b s}= & \hat{\Gamma}_{+} \otimes \frac{1}{2}\left[\hat{A}_{00}-\hat{A}_{++}\right]+\hat{\Gamma}_{-} \otimes \frac{1}{2}\left[\hat{A}_{00}-\hat{A}_{--}\right] \\
& +\hat{\Gamma}_{+-} \otimes \hat{A}_{+-}+\hat{\Gamma}_{-+} \otimes \hat{A}_{-+}+\hat{\Gamma}_{+0} \otimes \hat{A}_{+0} \\
& +\hat{\Gamma}_{0+} \otimes \hat{A}_{0+}+\hat{\Gamma}_{-0} \otimes \hat{A}_{-0}+\hat{\Gamma}_{0-} \otimes \hat{A}_{0-},
\end{aligned}
$$

where

$$
\begin{aligned}
\hat{\Gamma}_{+}=- & {\left[\cos ^{2}(\phi)-\sin ^{2}(\theta) \sin ^{2}(\phi)\right] \hat{\Gamma}_{P} } \\
- & {\left[\cos ^{2}(\phi)-\cos ^{2}(\theta) \sin ^{2}(\phi)\right] \hat{\Gamma}_{S}, } \\
\hat{\Gamma}_{-}=- & {\left[\sin ^{2}(\phi)-\sin ^{2}(\theta) \cos ^{2}(\phi)\right] \hat{\Gamma}_{P} } \\
& -\left[\sin ^{2}(\phi)-\cos ^{2}(\theta) \cos ^{2}(\phi)\right] \hat{\Gamma}_{S}, \\
\hat{\Gamma}_{+-}= & \hat{\Gamma}_{-+}=-\frac{1}{2} \sin (\phi) \cos (\phi)\left\{\left[1+\sin ^{2}(\theta)\right] \hat{\Gamma}_{P}\right. \\
& \left.+\left[1+\cos ^{2}(\theta)\right] \hat{\Gamma}_{S}\right\}, \\
\hat{\Gamma}_{+0}= & \hat{\Gamma}_{0+}=-\frac{1}{2} \sin (\theta) \cos (\theta) \sin (\phi)\left(\hat{\Gamma}_{P}-\hat{\Gamma}_{S}\right), \\
\hat{\Gamma}_{-0}= & \hat{\Gamma}_{0-}=-\frac{1}{2} \sin (\theta) \cos (\theta) \cos (\phi)\left(\hat{\Gamma}_{P}-\hat{\Gamma}_{S}\right) .
\end{aligned}
$$

Following the procedure outlined in the Appendix, then lead to a QME of the same form as Eq. (21), except for the fact that the field-independent dissipative term, $\mathcal{L}_{D}^{0}$, is now replaced by the following, more general, field-dependent dissipative term:

$$
\mathcal{L}_{D}\left(\hat{\rho}_{s}\right)=\sum_{i, j, k, l} \gamma_{i j k l}\left[\hat{A}_{i j}, \hat{A}_{k l} \hat{\rho}_{s}\right]+C . C .,
$$

where C.C. stands for "complex conjugate," $i, j, k, l=+$, $0,-$,

$$
\gamma_{i j k l}=\frac{1}{2 \hbar^{2}} \widetilde{C}_{i j k l}\left(-\omega_{k l}\right),
$$

$\omega_{k l}=\left(\omega_{k}-\omega_{l}\right) / 2[\mathrm{cf} . \mathrm{Eq} .(8)]$, and

$$
C_{i j k l}(\tau)=\operatorname{Tr}\left[\hat{\rho}_{b}^{\mathrm{eq}} e^{i \hat{H}_{b} t / \hbar} \hat{\Gamma}_{i j} e^{-i \hat{H}_{b} t / \hbar} \hat{\Gamma}_{k l}\right]
$$

[also note that $\hat{\Gamma}_{k k} \equiv \hat{\Gamma}_{k}$ ]. Explicit expressions for the rate coefficients $\left\{\gamma_{i j k l}\right\}$ in terms of the correlation function of the original bath operators, $\hat{\Gamma}_{P}$ and $\hat{\Gamma}_{S}$, are given in Tables IIIV, for the cases of correlated, uncorrelated, and anticorrelated dephasing, respectively.

It is important to emphasize that the validity of the new dissipative term in Eq. (32) is not restricted to weak driving fields. Furthermore, one expects Eq. (32) to reduce to its weak field counterpart, Eq. (22), in the limit of weak driving 
TABLE III. Same as Table II for uncorrelated dephasing $\left[C_{P S}(\tau)=0\right]$.

\begin{tabular}{|c|c|c|c|}
\hline & ++ & -- &,+--+ \\
\hline $\begin{array}{l}-- \\
+- \\
-+ \\
+0 \\
0+ \\
-0 \\
0-\end{array}$ & $\begin{array}{c}\frac{1}{2}\left[\left(c_{\phi}^{2}-s_{\theta}^{2} s_{\phi}^{2}\right)^{2} \widetilde{C}_{P}(0)\right. \\
\left.+\left(c_{\phi}^{2}-c_{\theta}^{2} s_{\phi}^{2}\right)^{2} \widetilde{C}_{S}(0)\right] \\
\frac{1}{2}\left[\left(c_{\phi}^{2}-s_{\theta}^{2} s_{\phi}^{2}\right)\left(s_{\phi}^{2}-s_{\theta}^{2} c_{\phi}^{2}\right)\right. \\
\widetilde{C}_{P}(0)+\left(c_{\phi}^{2}-c_{\theta}^{2} s_{\phi}^{2}\right) \\
\left.\left(s_{\phi}^{2}-c_{\theta}^{2} c_{\phi}^{2}\right) \widetilde{C}_{S}(0)\right] \\
\frac{1}{4} c_{\phi} s_{\phi}\left[\left(1+s_{\theta}^{2}\right)\left(c_{\phi}^{2}-s_{\theta}^{2} s_{\phi}^{2}\right)\right. \\
\widetilde{C}_{P}(0)+\left(1+c_{\theta}^{2}\right) \\
\left.\left(c_{\phi}^{2}-c_{\theta}^{2} s_{\phi}^{2}\right) \widetilde{C}_{S}(0)\right] \\
\frac{1}{4} c_{\theta} s_{\theta} s_{\phi}\left[\left(c_{\phi}^{2}-s_{\theta}^{2} s_{\phi}^{2}\right) \widetilde{C}_{P}(0)\right. \\
\left.-\left(c_{\phi}^{2}-c_{\theta}^{2} s_{\phi}^{2}\right) \widetilde{C}_{S}(0)\right] \\
\frac{1}{4} c_{\theta} s_{\theta} c_{\phi}\left[\left(c_{\phi}^{2}-s_{\theta}^{2} s_{\phi}^{2}\right) \widetilde{C}_{P}(0)\right. \\
\left.-\left(c_{\phi}^{2}-c_{\theta}^{2} s_{\phi}^{2}\right) \widetilde{C}_{S}(0)\right] \\
+0,0+ \\
\frac{1}{4} c_{\theta} s_{\theta} s_{\phi}\left[\left(c_{\phi}^{2}-s_{\theta}^{2} s_{\phi}^{2}\right) \widetilde{C}_{P}\left(\mp \nu_{+}\right)\right. \\
\left.-\left(c_{\phi}^{2}-c_{\theta}^{2} s_{\phi}^{2}\right) \widetilde{C}_{S}\left(\mp \nu_{+}\right)\right] \\
\frac{1}{4} c_{\theta} s_{\theta} s_{\phi}\left[\left(s_{\phi}^{2}-s_{\theta}^{2} c_{\phi}^{2}\right) \widetilde{C}_{P}\left(\mp \nu_{+}\right)\right. \\
\left.-\left(s_{\phi}^{2}-c_{\theta}^{2} c_{\phi}^{2}\right) \widetilde{C}_{S}\left(\mp \nu_{+}\right)\right] \\
\frac{1}{8} c_{\theta} s_{\theta} c_{\phi} s_{\phi}^{2}\left[\left(1+s_{\theta}^{2}\right) \widetilde{C}_{P}\left(\mp \nu_{+}\right)\right. \\
\left.-\left(1+c_{\theta}^{2}\right) \widetilde{C}_{S}\left(\mp \nu_{+}\right)\right] \\
\frac{1}{8} c_{\theta}^{2} s_{\theta}^{2} s_{\phi}^{2}\left[\widetilde{C}_{P}\left(\mp \nu_{+}\right)\right. \\
\left.+\widetilde{C}_{S}\left(\mp \nu_{+}\right)\right] \\
\frac{1}{8} c_{\theta}^{2} s_{\theta}^{2} c_{\phi} s_{\phi}\left[\widetilde{C}_{P}\left(\mp \nu_{+}\right)\right. \\
\left.+\widetilde{C}_{S}\left(\mp \nu_{+}\right)\right] \\
\end{array}$ & 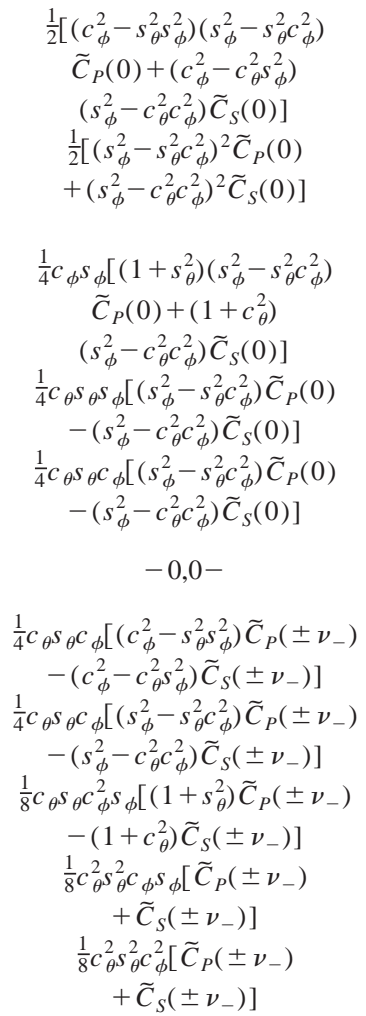 & $\begin{array}{c}\frac{1}{4} c_{\phi} s_{\phi}\left[\left(1+s_{\theta}^{2}\right)\left(c_{\phi}^{2}-s_{\theta}^{2} s_{\phi}^{2}\right)\right. \\
\widetilde{C}_{P}\left(\mp \nu_{+} \mp \nu_{-}\right)+\left(1+c_{\theta}^{2}\right) \\
\left.\left(c_{\phi}^{2}-c_{\theta}^{2} s_{\phi}^{2}\right) \widetilde{C}_{S}\left(\mp \nu_{+} \mp \nu_{-}\right)\right] \\
\frac{1}{4} c_{\theta} s_{\theta} c_{\phi}\left[\left(1+s_{\theta}^{2}\right)\left(s_{\phi}^{2}-s_{\theta}^{2} c_{\phi}^{2}\right)\right. \\
\widetilde{C}_{P}\left(\mp \nu_{+} \mp \nu_{-}\right)+\left(1+c_{\theta}^{2}\right) \\
\left.\left(s_{\phi}^{2}-c_{\theta}^{2} c_{\phi}^{2}\right) \widetilde{C}_{S}\left(\mp \nu_{+} \mp \nu_{-}\right)\right] \\
\frac{1}{8} c_{\phi}^{2} s_{\phi}^{2}\left[\left(1+s_{\theta}^{2}\right)^{2} \widetilde{C}_{P}\left(\mp \nu_{+} \mp \nu_{-}\right)\right. \\
\left.+\left(1+c_{\theta}^{2}\right)^{2} \widetilde{C}_{S}\left(\mp \nu_{+} \mp \nu_{-}\right)\right] \\
\\
\frac{1}{8} c_{\theta} s_{\theta} c_{\phi} s_{\phi}^{2}\left[\left(1+s_{\theta}^{2}\right) \widetilde{C}_{P}\left(\mp \nu_{+} \mp \nu_{-}\right)\right. \\
\left.-\left(1+c_{\theta}^{2}\right) \widetilde{C}_{S}\left(\mp \nu_{+} \mp \nu_{-}\right)\right] \\
\frac{1}{8} c_{\theta} s_{\theta} c_{\phi}^{2} s_{\phi}\left[\left(1+s_{\theta}^{2}\right) \widetilde{C}_{P}\left(\mp \nu_{+} \mp \nu_{-}\right)\right. \\
\left.-\left(1+c_{\theta}^{2}\right) \widetilde{C}_{S}\left(\mp \nu_{+} \mp \nu_{-}\right)\right]\end{array}$ \\
\hline
\end{tabular}

fields, i.e., when $\Omega_{P} \tau_{c}, \Omega_{S} \tau_{c} \ll 1 .^{51,52}$ Importantly, the dissipative term in Eq. (32) becomes explicitly field-dependent when we venture beyond this limit. This dependence comes about through $\theta, \phi$, and $\omega_{k l}$. The latter is of particular importance, due to the occurrence of $\widetilde{C}\left( \pm \nu_{+}\right), \widetilde{C}\left( \pm \nu_{-}\right)$, and $\widetilde{C}\left( \pm \nu_{+} \pm \nu_{-}\right)\left(\mathrm{cf}\right.$. Tables II-IV, and note that $\nu_{-}=\omega_{0-} / 2$ and $\left.\nu_{+}=\omega_{+0} / 2\right)$. Close to resonance $(\Delta \approx 0)$, the terms $\widetilde{C}$ $\left( \pm \nu_{+}\right), \widetilde{C}\left( \pm \nu_{-}\right)$, and $\widetilde{C}\left( \pm \nu_{+} \pm \nu_{-}\right)$all reduce to $\widetilde{C}(0)$ in the weak field limit $\left(\Omega_{P} \tau_{c}, \Omega_{S} \tau_{c} \ll 1\right)$, which is why Eq.
(22) only involves $\widetilde{C}(0)$. However, this is no longer true when $\Omega_{P}$ and $\Omega_{S}$ become comparable, and even larger than $\tau_{c}^{-1}$. In fact, $\widetilde{C}\left( \pm \nu_{+}\right), \widetilde{C}\left( \pm \nu_{-}\right)$, and $\widetilde{C}\left( \pm \nu_{+} \pm \nu_{-}\right)$all vanish when $\Omega_{P} \tau_{c}, \Omega_{S} \tau_{c} \gg 1$, which can lead to a dramatic field-induced suppression of dephasing. Thus, the driving fields provide a potentially valuable control over dephasing, and intense fields can be used to suppress dephasing in systems undergoing STIRAP in solution.

Numerical simulations based on Eq. (32) have been car-

TABLE IV. Same as Table II for anti-correlated dephasing $\left[C_{S}(\tau)=C_{P}(\tau)=-C_{P S}(\tau)=C(\tau)\right]$.

\begin{tabular}{lccc}
\hline \hline & ++ &,+--+ \\
\hline++ & $\frac{1}{2}\left(c_{\theta}^{2}-s_{\theta}^{2}\right)^{2} s_{\phi}^{4} \widetilde{C}(0)$ & $\frac{1}{2}\left(c_{\theta}^{2}-s_{\theta}^{2}\right)^{2} c_{\phi}^{2} s_{\phi}^{2} \widetilde{C}(0)$ & $-\frac{1}{4}\left(c_{\theta}^{2}-s_{\theta}^{2}\right)^{2} c_{\phi} s_{\phi}^{3} \widetilde{C}\left(\mp \nu_{+} \mp \nu_{-}\right)$ \\
-- & $\frac{1}{2}\left(c_{\theta}^{2}-s_{\theta}^{2}\right)^{2} c_{\phi}^{2} s_{\phi}^{2} \widetilde{C}(0)$ & $\frac{1}{2}\left(c_{\theta}^{2}-s_{\theta}^{2}\right)^{2} c_{\phi}^{4} \widetilde{C}(0)$ & $-\frac{1}{4}\left(c_{\theta}^{2}-s_{\theta}^{2}\right)^{2} c_{\phi}^{3} s_{\phi} \widetilde{C}\left(\mp \nu_{+} \mp \nu_{-}\right)$ \\
+- & $-\frac{1}{4}\left(c_{\theta}^{2}-s_{\theta}^{2}\right)^{2} c_{\phi} s_{\phi}^{3} \widetilde{C}(0)$ & $-\frac{1}{4}\left(c_{\theta}^{2}-s_{\theta}^{2}\right)^{2} c_{\phi}^{3} s_{\phi} \widetilde{C}(0)$ & $\frac{1}{8}\left(c_{\theta}^{2}-s_{\theta}^{2}\right)^{2} c_{\phi}^{2} s_{\phi}^{2} \widetilde{C}\left(\mp \nu_{+} \mp \nu_{-}\right)$ \\
-+ & $\frac{1}{2}\left(c_{\theta}^{2}-s_{\theta}^{2}\right) c_{\theta} s_{\theta} s_{\phi}^{3} \widetilde{C}(0)$ & $\frac{1}{2}\left(c_{\theta}^{2}-s_{\theta}^{2}\right) s_{\theta} c_{\theta} s_{\phi} c_{\phi}^{2} \widetilde{C}(0)$ & $\frac{1}{4}\left(s_{\theta}^{2}-c_{\theta}^{2}\right) c_{\theta} s_{\theta} c_{\phi} s_{\phi}^{2} \widetilde{C}\left(\mp \nu_{+} \mp \nu_{-}\right)$ \\
+0 & $\frac{1}{2}\left(c_{\theta}^{2}-s_{\theta}^{2}\right) c_{\theta} s_{\theta} c_{\phi} s_{\phi}^{2} \widetilde{C}(0)$ & $\frac{1}{2}\left(c_{\theta}^{2}-s_{\theta}^{2}\right) c_{\theta} s_{\theta} c_{\phi}^{3} \widetilde{C}(0)$ & $\frac{1}{4}\left(s_{\theta}^{2}-c_{\theta}^{2}\right) c_{\theta} s_{\theta} c_{\phi}^{2} s_{\phi} \widetilde{C}\left(\mp \nu_{+} \mp \nu_{-}\right)$ \\
$0+$ & $-0,0-$ & \\
-0 & $+0,0+$ & & \\
$0-$ & $\frac{1}{2}\left(c_{\theta}^{2}-s_{\theta}^{2}\right) c_{\theta} s_{\theta} c_{\phi} s_{\phi}^{2} \widetilde{C}\left( \pm \nu_{-}\right)$ & \\
++ & $\frac{1}{2}\left(c_{\theta}^{2}-s_{\theta}^{2}\right) c_{\theta} s_{\theta} s_{\phi}^{3} \widetilde{C}\left(\mp \nu_{+}\right)$ & $\frac{1}{2}\left(c_{\theta}^{2}-s_{\theta}^{2}\right) c_{\theta} s_{\theta} c_{\phi}^{3} \widetilde{C}\left( \pm \nu_{-}\right)$ & \\
-- & $\frac{1}{2}\left(c_{\theta}^{2}-s_{\theta}^{2}\right) c_{\theta} s_{\theta} c_{\phi}^{2} s_{\phi} \widetilde{C}\left(\mp \nu_{+}\right)$ & $\frac{1}{4}\left(s_{\theta}^{2}-c_{\theta}^{2}\right) c_{\theta} s_{\theta} c_{\phi}^{2} s_{\phi} \widetilde{C}\left( \pm \nu_{-}\right)$ & \\
+- & $\frac{1}{4}\left(s_{\theta}^{2}-c_{\theta}^{2}\right) c_{\theta} s_{\theta} c_{\phi} s_{\phi}^{2} \widetilde{C}\left(\mp \nu_{+}\right)$ & $\frac{1}{2} c_{\theta}^{2} s_{\theta}^{2} c_{\phi} s_{\phi} \widetilde{C}\left( \pm \nu_{-}\right)$ & \\
-+ & $\frac{1}{2} c_{\theta}^{2} s_{\theta}^{2} s_{\phi}^{2} \widetilde{C}\left(\mp \nu_{+}\right)$ & $\frac{1}{2} c_{\theta}^{2} s_{\theta}^{2} c_{\phi}^{2} \widetilde{C}\left( \pm \nu_{-}\right)$ & \\
+0 & & & \\
$0+$ & & & \\
-0 & & & \\
$0-$ & & &
\end{tabular}




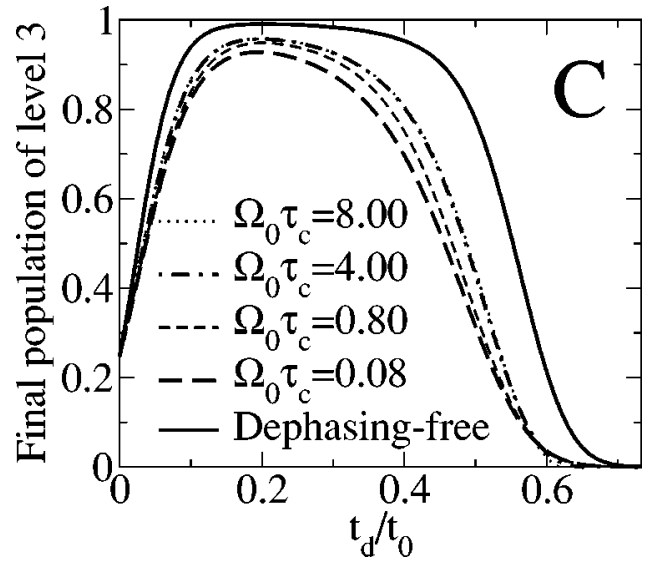

FIG. 8. The final value of $P_{3}^{r}$ as a function of the time delay between the Stokes and pump pulses, under field-dependent correlated pure dephasing, for different value of $\Omega_{0} \tau_{c}$. The dephasing-free results are also shown for reference. The parameters used in the simulation are given in the text.

ried out in order to gain further insight into the interplay between the driving fields and dephasing rate. The model and parameters used are the same as in Sec. IV. The bath correlation function was assumed to decay exponentially

$$
C(\tau)=C(0) e^{-|\tau| / \tau_{c}},
$$

such that

$$
\widetilde{C}(\omega)=\widetilde{C}(0) \frac{1}{1+\left(\omega \tau_{c}\right)^{2}},
$$

where, as before, $\widetilde{C}(0) t_{0}=2 C(0) \tau_{c} t_{0}=96.0 \hbar^{2}$. It should be noted that $\widetilde{C}(\omega) \rightarrow \widetilde{C}(0)$ when $\omega \tau_{c} \ll 1$, and that $\widetilde{C}(\omega) \rightarrow 0$ when $\omega \tau_{c} \gg 1$ (which correspond to the limits of weak and strong driving fields in our case).

Figures 8-10 show the final value of $P_{3}^{r}$ as a function of the time delay between the Stokes and pump pulses, $t_{d}$, as obtained from simulations of STIRAP under correlated (C), uncorrelated (U), and anti-correlated (A) dephasing, respectively. In each figure, we show the results obtained for different values of $\Omega_{0} \tau_{c}$. It should be noted that $\widetilde{C}(0)$ is held fixed, such that the dephasing term in the QME depends on $\Omega_{0} \tau_{c}$, rather than separately on $\Omega_{0}$ and $\tau_{c}$. Thus, increasing $\Omega_{0}$ (which is controllable) has the same effect as increasing

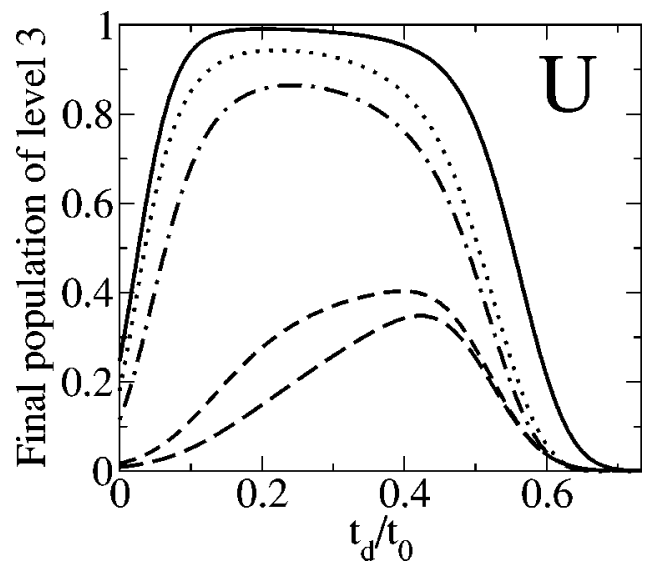

FIG. 9. Same as Fig. 8, under uncorrelated dephasing.

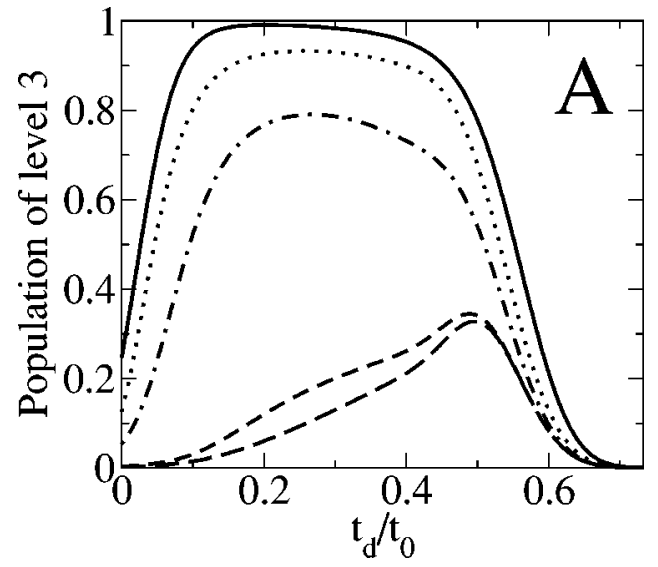

FIG. 10. Same as Fig. 8, under anti-correlated dephasing.

$\tau_{c}$ (which is usually not controllable in actual experiments). Increasing $\Omega_{0}$ instead of $\tau_{c}$ would actually also slightly modify the Hamiltonian term, such that it better satisfies the adiabaticity criterion, $\Omega_{0} t_{d} \gg 1$. However, by using the same value of $\Omega_{0}$ as in Sec. IV $\left(\Omega_{0} t_{0}=240.0\right)$ we insure that this effect is negligible.

Figures 8-10 show an improvement in the STIRAP efficiency and robustness as $\Omega_{0} \tau_{c}$ is increased (which could be realized in practice by employing more intense fields). The improvement is rather modest in the case of correlated dephasing, where the efficiency is high even when the dephasing is field-independent. However, the improvement is dramatic in the cases of uncorrelated and anti-correlated dephasing. In those cases, the transfer efficiency rapidly increases from $35 \%$ and $33 \%$ when $\Omega_{0} \tau_{c}=0.08$, to $95 \%$ and 93\%, respectively, when $\Omega_{0} \tau_{c}=8.0$.

Figures 11-13 show the time evolution of $P_{1}^{r}, P_{2}^{r}, P_{3}^{r}$, $\operatorname{Im} P_{12}^{r}, \operatorname{Im} P_{23}^{r}$, and $\operatorname{Re} P_{13}^{r}$ under correlated, uncorrelated and anti-correlated dephasing, respectively, at $\Omega_{0} \tau_{c}=4.0$, and for a specific time delay $\left(t_{d} / t_{0}=1 / 3\right)$. It is instructive to

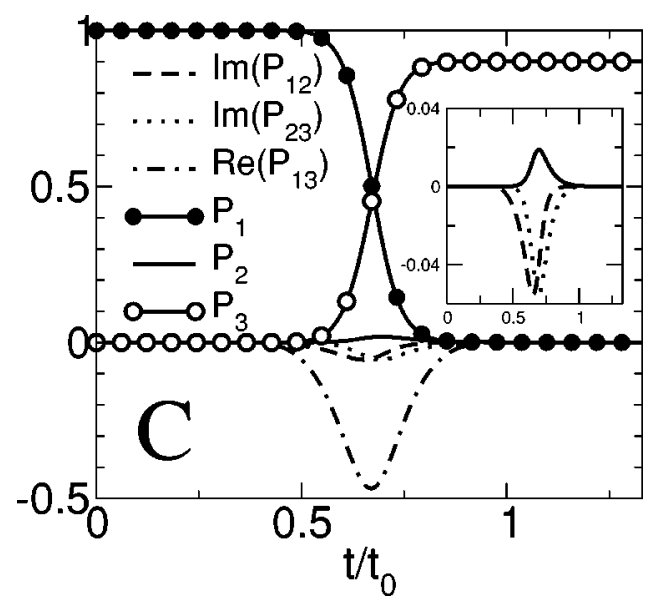

FIG. 11. The time evolution of $P_{1}^{r}, P_{2}^{r}, P_{3}^{r}, \operatorname{Im} P_{12}^{r}, \operatorname{Im} P_{23}^{r}$, and $\operatorname{Re} P_{13}^{r}$ throughout the STIRAP process, at $\Omega_{0} \tau_{c}=4.0$, for $t_{d} / t_{0}=1 / 3$, under fielddependent correlated dephasing. The Stokes (pump) pulse is turned on at $t / t_{0}=0\left(t / t_{0}=1 / 3\right)$, peaks at $t / t_{0}=1 / 2\left(t / t_{0}=5 / 6\right)$ and is turned off at $t / t_{0}$ $=1\left(t / t_{0}=4 / 3\right)$. Note that $P_{13}^{r}$ is purely real, while $P_{12}^{r}$ and $P_{23}^{r}$ are purely imaginary throughout the dynamics. The inset shows a close-up of $P_{2}^{r}, P_{12}^{r}$, and $P_{23}^{r}$. 


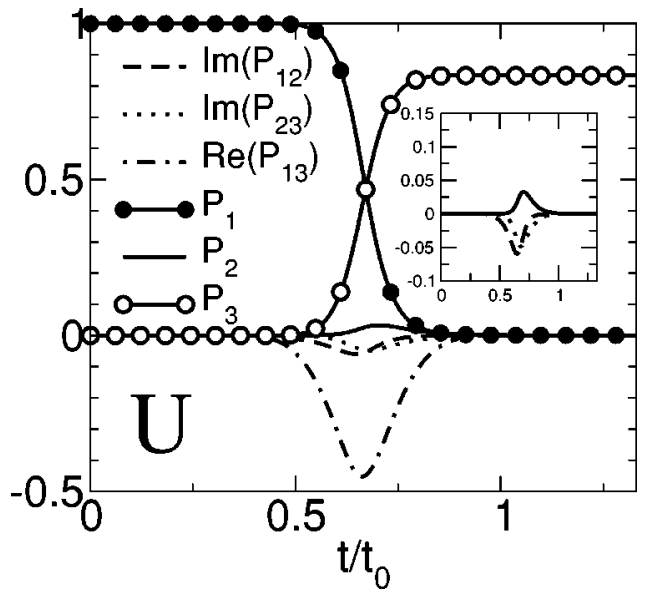

FIG. 12. Same as Fig. 11, for field-dependent uncorrelated dephasing.

compare these figures with Figs. 5-7 which show the predicated temporal behavior under the same conditions, based on the weak field treatment. In the case of correlated dephasing, we see that the improved STIRAP efficiency is mostly due to weakening of the nonadiabatic population transfer to level 2 (cf. insets in Figs. 5 and 11), which is in turn due to the weaker dephasing. On the other hand, the improved STIRAP efficiency in the cases of uncorrelated and anti-correlated dephasing is primarily due to field-induced suppression of the dephasing of $P_{13}$, as indicated by the buildup of $P_{13}$ in Figs. 12 and 13, which is comparable to that in the dephasing-free case. The diminished dephasing of $P_{12}$ and $P_{23}$ also weaken the nonadiabatic transitions to level 2, which provide further improvement to the STIRAP efficiency in those cases. (Compare insets in Figs. 6 and 7 to those in Figs. 12 and 13, respectively.)

\section{RELATIONSHIP TO OTHER STUDIES}

In this section, we discuss the relationship of the results presented in this paper to these in two other recent studies that considered the influence of decoherence on STIRAP. ${ }^{30,50}$

In Ref. 30, Demirplak and Rice have considered the prospect of using STIRAP in liquid solutions. Their basic model is similar to ours, except for the fact that the bath is

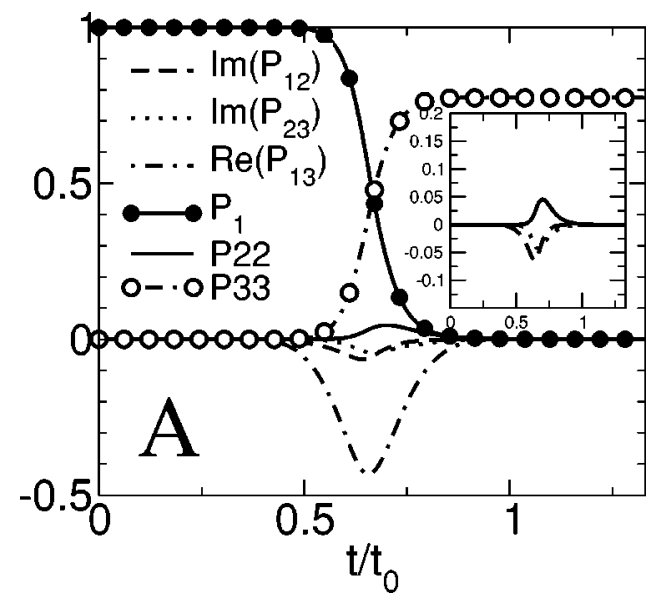

FIG. 13. Same as Fig. 11, for field-dependent anti-correlated dephasing. assumed classical, such that the quantum-mechanical bath operators $\hat{\Gamma}_{P}$ and $\hat{\Gamma}_{S}$ may be replaced by fluctuating classical random variables, $\Gamma_{P}(t)$ and $\Gamma_{S}(t)$. The fluctuations of the classical stochastic forces $\Gamma_{P}(t)$ and $\Gamma_{S}(t)$ were then assumed to be uncorrelated, and to follow Gaussian statistics, with a zero first moment and an exponentially decaying correlation function. Demirplak and Rice performed stochastic simulations within the framework of this model, which involved extensive averaging over solutions of the Schrödinger equation for the STIRAP system, obtained for different histories of the bath fluctuations (it should be noted that the classical nature of the bath fluctuations allows for a description in terms of a wave function for each realization of the noise history, followed by ensemble averaging over those histories). It should be emphasized that such an approach is not computationally feasible in our case, because of the quantum-mechanical treatment of the bath. As a result, one has to resort to an alternative approach in the quantum case, namely one that describes the system in terms of a density operator and the dephasing in terms of a QME. The advantage of the QME-based approach lies in its ability to avoid the extensive averaging over histories of bath fluctuations, which makes it more economical and easier to interpret. Its major disadvantage has to do with the fact that the quantum dynamics described by the QME is not equivalent to the quantum analogue of the classical bath model of Ref. 30, since it involves the additional assumptions of weak systembath coupling and Markovity.

The results presented in Ref. 30 were obtained for different values of the correlation time of $\Gamma_{P}(t)$ and $\Gamma_{S}(t)$, which is analogous to our $\tau_{c}$, and at a fixed value of the peak Rabi frequency. It was found that the STIRAP efficiency approaches its dephasing-free value in the two opposite limits of $\tau_{c} \gg t_{d}$ and $\tau_{c} \ll t_{d}$ [cf. Fig. 2 in Ref. 30]. It should be noted that our analysis assumes at the outset that $\tau_{c} \ll t_{d}$. Furthermore, $\tau_{c} \gg t_{d}$ implies that the energy levels do not fluctuate on the time scale of the experiment, and hence that the system is not subject to dephasing. It is interesting to note that the system is subject to inhomogeneous broadening in this case, which would lead to deviations from the twophoton resonance condition and result in a lower STIRAP efficiency. However, the Gaussian distribution of $\Gamma_{P}(t)$ and $\Gamma_{S}(t)$ was assumed to be very narrow in comparison to the Rabi frequency in Ref. 30, which made this effect negligible. The opposite limit, $\tau_{c} \ll t_{d}$, corresponds to that of motional narrowing, where the dephasing rate constant essentially diminishes when $\tau_{c}$ is decreased. It should be noted that $\widetilde{C}(0)=2 C(0) \tau_{c}$, when the bath correlation function is exponential, and that $C(0)$, rather than $\widetilde{C}(0)$, is held fixed in Ref. 30. Thus, in this case, decreasing $\tau_{c}$ essentially suppresses the dephasing rate (note however that, in practice, $\tau_{c}$ is usually not a controllable quantity). In our case, $\widetilde{C}(0)$ is held fixed at a value that corresponds to significant fieldindependent dephasing, in order to avoid this possibility and focus on the dependence upon the field intensity, which is a controllable quantity. Finally, we note that the STIRAP efficiency in situations where $\Omega_{0} \tau_{c}>1$ [cf. Figs. 2(c)-2(f) in Ref. 30] is relatively high, either because the dephasing is 
suppressed by the field when $\Omega_{0} \tau_{c}>1$ [plots 2(c) and 2(d) there], or simply because $\tau_{c} \gg t_{d}$ [plots 2(e) and 2(f) there].

In Ref. 50, Bergmann and co-workers have considered the effect of phase noise in the driving fields, on the STIRAP efficiency, using a model which is very similar to that in Ref. 30 . The great similarity between those two papers originates from the fact that phase noise in the Stokes and pump lasers leads to fluctuations in the diagonal elements of the system Hamiltonian, and is therefore mathematically equivalent to the description of solvent-induced dephasing in terms of classical bath fluctuations, as in Ref. 30. Here too, the fluctuations were assumed to follow exponentially correlated Gaussian statistics, although different degree of correlation between the dephasing in the Stokes and pump transitions were considered (uncorrelated, as in Ref. 30, as well as correlated and partially correlated). The authors of Ref. 50 presented results that show the dependence of the STIRAP efficiency on the Rabi frequency of the driving fields, up to relatively high values of the latter. In the case of uncorrelated dephasing, they found that the STIRAP efficiency approaches its noise-free value when $\Omega_{0} \tau_{c}>5$, while in the case of correlated dephasing, they found that the STIRAP efficiency is very close to the noise-free value even for weak fields. Those results are consistent with the observations based on the QME-based approach, which were reported in Secs. IV and V of the present paper. Interestingly, the difference between a description in terms of explicitly fluctuating frequency modulations, as opposed to relaxation rate constants in a QME (the present work), gave rise to seemingly different interpretations of those behaviors. For example, the authors of Ref. 50 attributed the reduced efficiency in the uncorrelated weak-field regime to detuning away from the two-photon resonance condition, while we attribute the same effect to dephasing within the $\{|1\rangle,|3\rangle\}$ subspace (cf. Fig. 2). However, those two interpretations represent the same physical phenomenon as viewed from either the frequency domain point of view (detuning and line broadening), or the time domain point of view (dephasing). Similarly, the slight decrease of the efficiency in the correlated case has been attributed to enhanced nonadiabaticity due to dynamical detuning away from the one-photon resonance condition in Ref. 50, which is the frequency domain analogue of what we described as dephasing-enhanced nonadiabaticity, from a time domain perspective.

We would also like to draw attention to the fact that the general idea of utilizing intense driving fields to suppress dephasing is not new, and has been suggested and demonstrated in the past, in different contexts. Early work in this general area has been motivated by the observation of deviations from the standard Bloch equations in magnetic resonance experiments, and later on in optical spectroscopy (cf. Ref. 51 and references therein). Similar ideas have resurfaced more recently in the literature on decoherence, and particularly in the context of quantum computing, ${ }^{57}$ and ion traps. ${ }^{58-60}$

\section{CONCLUSIONS}

STIRAP is a general and versatile technique for controlling population transfer. In this paper we considered the prospects of applying it under the influence of pure dephasing. In the limit of weak driving fields $\left(\Omega \tau_{c} \ll 1\right)$, we found that the rate of pure dephasing is field-independent, and tends to lower the population transfer efficiency. The actual reduction in efficiency increases with the ability of the bath fluctuations to directly dephase $P_{13}^{r}$ (the coherence that corresponds to the transition between the initial and final states). Further reduction in the efficiency occurs due to dephasing enhanced nonadiabaticity, which results from symmetry breaking in the buildups of $P_{23}^{r}$ and $P_{12}^{r}$ (the coherences that correspond to the Stokes and pump transitions, respectively). The net result is a rather poor efficiency, except in the case of correlated dephasing, where $P_{13}^{r}$ is not subject to direct pure dephasing.

A more encouraging picture emerges once we venture beyond the limit of weak driving fields. The dissipative term in the QME becomes explicitly field-dependent in this case, thereby turning the dephasing rate into a controllable quantity. In fact, we have found that pure dephasing can be effectively suppressed when the Rabi frequency, $\Omega$, becomes larger than the inverse bath correlation time, $\tau_{c}^{-1}$. More specifically, we have shown that the STIRAP efficiency becomes comparable to its dephasing-free value, and regains it relative insensitivity to the value of the time delay between the Stokes and pump pulses, when $\Omega \tau_{c}>4$, regardless of the particular dephasing mechanism. This rather remarkable result suggests that STIRAP may be feasible in condensedphase solutions.

It should be noted that efficient dephasing-free STIRAP already requires that $\Omega_{0} t_{d} \gg 1$, in order to secure adiabaticity. However, we have assumed that $t_{d} \sim t_{0}$ is larger than the pure dephasing life times, which are in turn assumed to be longer than the bath correlation time, $\tau_{c}$, in the derivation of the QME. In pulsed-laser-based dephasing-free STIRAP, $t_{d}$ $\sim$ ns, while $\tau_{c} \sim$ ps is a typical correlation time in room temperature liquid solutions. Thus realizing the conditions that allow for the suppression of pure dephasing would require that the Rabi frequency of the driving field is increased by a factor of $10^{3}$ relative to the minimum required by adiabaticity. However, it should be noted that Demirplak and Rice have recently argued that it may be feasible to use ps pulses in order to perform STIRAP in liquid solutions, ${ }^{30}$ in which case the condition for adiabaticity will essentially coincide with that required for the suppression of dephasing. It should also be noted that by increasing the field intensity, it becomes possible to satisfy adiabaticity with shorter pulses. However, using shorter pulses may invalidate our assumption that the variation of the pulse envelope is slow relative to $\tau_{c}$, and make it necessary to extend the theory so as to account for this scenario.

The experimental realization of the above scheme may be complicated by several factors: First, producing such intense light pulses on the ns time scale may be challenging. Second, applying such intense fields to a condensed phase sample may result in undesired secondary processes and/or radiation damage. Third, the assumption of weak systembath coupling will not always be valid (e.g., in the case of polar solute and solvent with pronounced solvation dynam- 
ics). A more detailed analysis of those issues will be the subject of future studies.

Finally, it should be noted that the STIRAP efficiency provides a rather unique and very sensitive probe of solventsolute interactions. For example, a high STIRAP efficiency in the limit of weak driving fields would be indicative of correlated dephasing, which implies that the Stokes and pump transitions are dephased by the same bath DOF. Alternatively, measuring the STIRAP efficiency as a function of the Rabi frequency, beyond the limit of weak driving fields, provides direct information on $\tau_{c}$, i.e., on the dynamics of the bath DOF which are coupled to the system.

\section{ACKNOWLEDGMENT}

This project was supported by the National Science Foundation FOCUS Center, through Grant No. PHY0114336

\section{APPENDIX A: THE QUANTUM MASTER EQUATION OF A DRIVEN SYSTEM}

In this Appendix, we outline the derivation of the QME in the case of a system which is driven by an explicitly time-dependent driving field.

Consider a system with an explicitly time dependent Hamiltonian, $\hat{H}_{s}(t)$, which is coupled to a bath, with the Hamiltonian $\hat{H}_{b}$, such that the overall Hamiltonian is

$$
\hat{H}=\hat{H}_{s}(t)+\hat{H}_{b}+\lambda \hat{H}_{b s} .
$$

Here, $\hat{H}_{b s}$ is the system-bath interaction term, and $\lambda$ is a coupling coefficient, which will be used in order to keep track of the order in the ensuing perturbation expansion. In the limit of weak system-bath coupling and Markovian dynamics, one can show that the reduced dynamics of the system is governed by the following QME: ${ }^{54,61,62}$

$$
\frac{d}{d t} \hat{\rho}_{s}(t)=-\frac{i}{\hbar} \mathcal{L}_{s}(t) \hat{\rho}_{s}(t)+\mathcal{L}_{D}(t) \hat{\rho}_{s}(t)
$$

where $\hat{\rho}_{s}(t)$ is the system's reduced density operator

$$
\mathcal{L}_{s}(t)=\left[\hat{H}_{s}(t), \cdot\right]
$$

represents the bath-free Hamiltonian contribution to the dynamics, and

$$
\begin{aligned}
\mathcal{L}_{D}(t)= & -\frac{\lambda^{2}}{\hbar^{2}} \int_{0}^{\infty} d \tau \operatorname{Tr}_{b}\left\{\mathcal{L}_{b s} e^{-i \mathcal{L}_{b} \tau / \hbar} T_{+}\left[e^{-i \int_{t-\tau}^{t} d t^{\prime} \mathcal{L}_{s}\left(t^{\prime}\right) / \hbar}\right]\right. \\
& \left.\times \mathcal{L}_{b s} \hat{\rho}_{b}^{\mathrm{eq}} T_{-}\left[e^{i \int_{t-\tau}^{t} d t^{\prime} \mathcal{L}_{s}\left(t^{\prime}\right) / \hbar}\right]\right\},
\end{aligned}
$$

is the dissipation super-operator. Here, $\mathcal{L}_{b s}=\left[\hat{H}_{b s}, \cdot\right], T_{ \pm}$ correspond to positive $(+)$, and negative $(-)$ time ordering, ${ }^{63}$ and

$$
\hat{\rho}_{b}^{\mathrm{eq}}=e^{-\beta \hat{H}_{b}} \operatorname{Tr}\left(e^{-\beta \hat{H}_{b}}\right) .
$$

It should be noted that in deriving Eq. (A4), we assumed an initial state of the form $\hat{\rho}_{s}(0) \otimes \hat{\rho}_{b}^{\mathrm{eq}}$ for the overall system. This choice does not limit the generality of the treatment, as it is always possible to alter the initial state via equilibration and/or use of the driving term in the system Hamiltonian.
For the sake of simplicity, let us restrict ourselves to a system-bath coupling of the form

$$
\hat{H}_{b s}=\hat{F} \otimes \hat{\Gamma},
$$

where $\hat{F}$ is a Hermitian system operator and $\hat{\Gamma}$ is a Hermitian bath operator (the following results can be extended in a straightforward manner to the more general case, where $\hat{H}_{b s}$ consists of a sum of such terms). We will also assume, for simplicity, that $\langle\hat{\Gamma}\rangle_{\mathrm{eq}}=\operatorname{Tr}\left(\hat{\rho}_{b}^{\mathrm{eq}} \hat{\Gamma}\right)=0\left(\langle\hat{\Gamma}\rangle_{\mathrm{eq}} \neq 0\right.$ would simply require that we substitute $\hat{H}_{s}(t)+\langle\hat{\Gamma}\rangle_{\mathrm{eq}} \hat{F}$ for $\hat{H}_{s}(t)$ in Eq. (A3), and use $\delta \hat{\Gamma}=\hat{\Gamma}-\langle\hat{\Gamma}\rangle_{\text {eq }}$ instead of $\hat{\Gamma}$ in the analysis that follows). Substitution of Eq. (A6) into Eq. (A4), followed by some algebra, then leads to the following result:

$$
\mathcal{L}_{D}(t) \hat{\rho}_{s}(t)=-\lambda^{2}\left[\hat{F}, \hat{R}(t) \hat{\rho}_{s}(t)-\left\{\hat{R}(t) \hat{\rho}_{s}(t)\right\}^{\dagger}\right]
$$

where

$$
\hat{R}(t)=\frac{1}{\hbar^{2}} \int_{0}^{\infty} d \tau C(\tau) T_{+}\left[e^{-i \int_{t-\tau}^{t} d t^{\prime} \mathcal{L}_{s}\left(t^{\prime}\right) / \hbar}\right] \hat{F}
$$

and

$$
C(\tau)=\operatorname{Tr}\left[\hat{\rho}_{b}^{\mathrm{eq}} e^{i \hat{H}_{b} t / \hbar} \hat{\Gamma} e^{-i \hat{H}_{b} t / \hbar} \hat{\Gamma}\right],
$$

is the equilibrium autocorrelation function of the bath operator, $\hat{\Gamma}$.

Two limits of Eq. (A7) are of particular importance for the present paper. The first limit corresponds to a system Hamiltonian of the form $\hat{H}_{s}(t)=\hat{H}_{s}^{0}+\lambda \hat{W}(t)$, where $\hat{H}_{s}^{0}$ is stationary and $\hat{W}(t)$ is explicitly time-dependent [e.g., $\hat{W}(t)$ may represent driving by laser light, when the latter is treated as a classical field]. It should be noted that the same coupling coefficient, $\lambda$, is used for $\hat{W}(t)$ and $\hat{H}_{b s}$, so as to enable us to treat the corresponding powers in the perturbation expansions on equal footing. We note that $\mathcal{L}_{D}$ in Eq. (A7) is already of second order in $\lambda$, which originates from treating the system-bath interaction term, $\lambda \hat{H}_{b s}$, as a small perturbation. To this, one may now add the assumption that the field-matter interaction term, $\lambda \hat{W}(t)$, can be treated as an equally small perturbation. One may then expand $T_{+}\left[e^{-i \int_{t-\tau}^{t} d t^{\prime} \mathcal{L}_{s}\left(t^{\prime}\right) / \hbar}\right]$ in powers of $\lambda$, and only keep the zeroorder term, such that

$$
\hat{R}(t) \stackrel{\hat{W}(t) \rightarrow 0}{\Rightarrow} \hat{R}_{w c}=\frac{1}{\hbar^{2}} \int_{0}^{\infty} d \tau C(\tau) e^{-i \mathcal{L}_{s}^{0} \tau / \hbar} \hat{F} .
$$

The substitution of $\hat{R}_{w c}$ in Eq. (A10) into Eq. (A7) then yields the exact same dissipative term that one would obtain in the case of field-free dissipation, i.e., when $\hat{H}_{S}=\hat{H}_{S}^{0}$ [the same result can also be obtained by starting out with an overall Hamiltonian of the form $\hat{H}=\hat{H}_{s}^{0}+\hat{H}_{b}+\lambda(\hat{W}(t)$ $\left.+\hat{H}_{b s}\right)$, and treating $\lambda\left(\hat{W}(t)+\hat{H}_{b s}\right)$, rather than just $\lambda \hat{H}_{b s}$, as a small perturbation, in the derivation of the QME]. However, it is important to emphasize that this result is an approximation which is only valid in the limit of weak driving fields. It should also be emphasized that the driving term still 
affects the reduced system dynamics through the Hamiltonian part of the QME, despite the fact that the dissipative part is independent of it in this limit.

The result in Eq. (A10) can be further simplified if one rewrites $\hat{F}$ in terms of the eigen-projectors of $\hat{H}_{s}^{0}$ :

$$
\hat{F}=\sum_{i, j} f_{i j}|i\rangle\langle j|,
$$

where $\hat{H}_{s}^{0}|i\rangle=E_{i}^{0}|i\rangle$. Upon substitution into Eq. (A10) this yields

$$
\hat{R}_{w c}=\sum_{i, j} f_{i j}|i\rangle\langle j|\left(\kappa_{j i}+i \delta_{j i}\right),
$$

where the rate constants, $\left\{\kappa_{j i}\right\}$, and Lamb shifts, $\left\{\delta_{j i}\right\}$, are given by the following standard expressions:

$$
\kappa_{i j}=\frac{1}{2 \hbar^{2}} \widetilde{C}\left(\omega_{j i}\right)
$$

and

$$
\delta_{j i}=\frac{1}{2 \pi \hbar^{2}} \mathcal{P} \int_{-\infty}^{\infty} d \omega \frac{\widetilde{C}(\omega)}{\omega-\omega_{j i}},
$$

with $\quad \omega_{j i}=-\omega_{i j}=\left(E_{j}^{0}-E_{i}^{0}\right) / \hbar \quad$ and $\quad \widetilde{C}(\omega)$ $=\int_{-\infty}^{\infty} d \tau C(\tau) e^{i \omega \tau}$. The Lamb shifts, $\left\{\delta_{i, j}\right\}$, make a purely imaginary contribution to Eq. (A12), and therefore amount to a slight shift in the system's Hamiltonian, which is often neglected. The relaxation coefficients, $\left\{\kappa_{j i}\right\}$, are real (since $\hat{\Gamma}$ is Hermitian), and lead to population and phase relaxation. Importantly, they also satisfy detailed balance:

$$
\frac{\kappa_{j i}}{\kappa_{i j}}=e^{\beta \hbar \omega_{j i}} .
$$

This weak field approach is applied to STIRAP in Sec. IV.

Another important limit of Eq. (A7), corresponds to the case of slowly varying $\hat{H}_{s}(t)$. More specifically if $\hat{H}_{s}(t)$ varies on a time scale which is much longer than the time scale on which $C(\tau)$ decays to zero (the so-called bath correlation time, $\tau_{c}$ ), then one can simplify $\hat{R}(t)$ in the following manner:

$$
\hat{R}(t) \stackrel{\text { slow }_{s}(t)}{\Rightarrow} \hat{R}_{\text {inst }}(t)=\frac{1}{\hbar^{2}} \int_{0}^{\infty} d \tau C(\tau) e^{-i \mathcal{L}_{s}(t) \tau / \hbar} \hat{F}
$$

It should be noted that $\hat{R}_{\text {inst }}(t)$ has the same form as in the case of a stationary system Hamiltonian, if one assumes that the latter can be substituted by the instantaneous $\hat{H}_{s}(t)$. One can also rewrite $\hat{F}$ in terms of the instantaneous eigenprojectors of $\hat{H}_{s}(t)$,

$$
\hat{F}=\sum_{i, j} f_{i j}(t)|i(t)\rangle\langle j(t)|,
$$

where $\hat{H}_{s}(t)|i(t)\rangle=E_{i}(t)|i(t)\rangle$. Upon substitution into Eq. (A16), this leads to an expression for $\hat{R}_{\text {inst }}(t)$ which is similar to Eq. (A12), except for the fact that $\omega_{j i}(t)=\left(E_{j}(t)\right.$ $\left.-E_{i}(t)\right) / \hbar,\left\{f_{i j}(t)\right\}$ and $|i(t)\rangle\langle j(t)|$ are now explicitly timedependent, and dictated by the variation of $\hat{H}_{s}(t)$ over time.
Thus, in the case of a slowly varying $\hat{H}_{s}(t), \mathcal{L}_{D}$ in the QME is designed so as to relax the system toward a "moving target," in the form of a Boltzmann equilibrium state which corresponds to the instantaneous system Hamiltonian. . $^{29,64-66}$

The relaxation dynamics in the case of moderate to strong coupling to rapidly varying driving fields, would generally require a direct numerical solution of the QME with the dissipative term in Eq. (A7). Included in this category are optical and infrared (IR) driving fields, where the period of the oscillating electromagnetic field is comparable to, or smaller than, the time scale of the fastest molecular motions. However, one may still bypass Eq. (A7) in cases involving circularly polarized fields, or when the RWA applies. This can be done by working in the RF, where the explicit timedependence of the effective Hamiltonian is only due to that of the pulse envelop. For nonultrafast pulses, where the variation of the envelop over time is slow relative to the bath correlation time, one can use the above-mentioned limit of slowly varying $\hat{H}_{s}(t)$, within the RF picture. The end result is a standard-looking QME for the reduced density operator in the RF, with the dissipative part dictated by the instantaneous effective RF system Hamiltonian [cf. Eq. (A16)]. This approach is applied to STIRAP in Sec. V.

${ }^{1}$ D. J. Tannor and S. A. Rice, Adv. Chem. Phys. 70, 441 (1988).

${ }^{2}$ R. J. Gordon and S. A. Rice, Annu. Rev. Phys. Chem. 48, 601 (1997).

${ }^{3}$ H. Rabitz and W. Zhu, Acc. Chem. Res. 33, 572 (2000).

${ }^{4}$ S. A. Rice and M. Zhao, Optical Control of Molecular Dynamics (Wiley, New York, 2000).

${ }^{5}$ S. A. Rice and S. P. Shah, Phys. Chem. Chem. Phys. 4, 1683 (2002).

${ }^{6} \mathrm{M}$. Shapiro and P. Brumer, Principles of the Quantum Control of Molecular Processes (Wiley, Hoboken, New Jersey, 2002).

${ }^{7}$ R. Kosloff et al., Chem. Phys. 139, 201 (1989).

${ }^{8}$ W. Zhu, J. Botina, and H. Rabitz, J. Chem. Phys. 108, 1953 (1998).

${ }^{9}$ Y. Ohtsuki, W. Zhu, and H. Rabitz, J. Chem. Phys. 110, 9825 (1999).

${ }^{10}$ A. Assion et al., Science 282, 919 (1998).

${ }^{11}$ R. J. Levis, G. M. Menkir, and H. Rabitz, Science 292, 709 (2001).

${ }^{12}$ B. J. Pearson, J. L. White, T. C. Weinacht, and P. H. Bucksbaum, Phys. Rev. A 63, 063412 (2001)

${ }^{13}$ F. Grossmann, T. Dittrich, P. Jung, and P. Hänggi, Phys. Rev. Lett. 67, 516 (1991).

${ }^{14}$ R. Bavli and H. Metiu, Phys. Rev. Lett. 69, 1986 (1992).

${ }^{15}$ D. G. Evans, R. D. Coalson, H. J. Kim, and Y. Dakhnovskii, Phys. Rev. Lett. 75, 3649 (1995).

${ }^{16}$ A. Bartana, R. Kosloff, and D. J. Tannor, J. Chem. Phys. 99, 196 (1993).

${ }^{17}$ A. Bartana, R. Kosloff, and D. J. Tannor, J. Chem. Phys. 106, 1435 (1997).

${ }^{18}$ D. E. Makarov and N. Makri, Phys. Rev. B 52, R2257 (1995).

${ }^{19}$ N. Makri, J. Chem. Phys. 106, 2286 (1997).

${ }^{20}$ N. Makri and L. Wei, Phys. Rev. E 55, 2475 (1997).

${ }^{21}$ R. I. Cukier, C. Denk, and M. Morillo, Chem. Phys. 217, 179 (1997).

${ }^{22}$ M. Grifoni and P. Hänggi, Phys. Rep. 304, 229 (1998).

${ }^{23}$ O. Kühn, Eur. Phys. J. D 6, 49 (1999).

${ }^{24}$ H. Naundorf, K. Sundermann, and O. Kühn, Chem. Phys. 240, 163 (1999)

${ }^{25}$ O. Kuhn, Y. Zhao, F. Shuang, and Y. Yan, J. Chem. Phys. 112, 6104 (2000).

${ }^{26}$ N. Dŏslić, O. Kühn, J. Manz, and K. Sundermann, J. Phys. Chem. A 102, 9645 (1998)

${ }^{27}$ N. Dóslić et al., Phys. Chem. Chem. Phys. 1, 1249 (1999).

${ }^{28}$ T. C. Weinacht, J. L. White, and P. H. Bucksbaum, J. Phys. Chem. A 103, 10166 (1999).

${ }^{29}$ E. Geva, J. Chem. Phys. 116, 1629 (2001).

${ }^{30}$ M. Demirplak and S. A. Rice, J. Chem. Phys. 116, 8028 (2002).

${ }^{31}$ Y. Ohtsuki, K. Nakagami, W. Zhu, and H. Rabitz, Chem. Phys. 287, 197 (2003).

${ }^{32}$ R. S. Judson and H. Rabitz, Phys. Rev. Lett. 68, 1500 (1992). 
${ }^{33}$ M. Q. Phan and H. Rabitz, Chem. Phys. 217, 389 (1997).

${ }^{34}$ C. J. Bardeen et al., Chem. Phys. Lett. 280, 151 (1997).

${ }^{35}$ M. Q. Phan and H. Rabitz, J. Chem. Phys. 110, 34 (1999).

${ }^{36}$ S. Vajda et al., Chem. Phys. 267, 231 (2001).

${ }^{37}$ T. Brixner, B. Kiefer, and G. Gerber, Chem. Phys. Lett. 267, 241 (2001).

${ }^{38}$ T. C. Weinacht et al., Chem. Phys. Lett. 344, 333 (2001).

${ }^{39}$ T. C. Weinacht and P. H. Bucksbaum, J. Opt. B: Quantum Semiclassical Opt. 4, R35 (2002).

${ }^{40}$ J. R. Kuklinski, U. Gaubatz, F. T. Hioe, and K. Bergmann, Phys. Rev. A 40, 6741 (1989).

${ }^{41}$ U. Gaubatz, P. Rudecki, S. Schiemann, and K. Bergmann, J. Chem. Phys. 92, 5363 (1990)

${ }^{42}$ A. Kühn et al., J. Chem. Phys. 96, 4215 (1992).

${ }^{43}$ C. E. Caroll and F. T. Hioe, Phys. Rev. A 42, 1522 (1990).

${ }^{44}$ T. A. Laine and S. Stenholm, Phys. Rev. A 53, 2501 (1996).

${ }^{45}$ N. V. Vitanov and S. Stenholm, Opt. Commun. 127, 215 (1996).

${ }^{46}$ M. P. Fewell, B. W. Shore, and K. Bergmann, Aust. J. Phys. 50, 281 (1997).

${ }^{47}$ A. Kühn, S. Steuerwald, and K. Bergmann, Eur. Phys. J. D 1, 57 (1998).

${ }^{48}$ K. Bergmann, H. Theuer, and W. Shore, Rev. Mod. Phys. 70, 1003 (1998).

${ }^{49}$ I. R. Sola, V. S. Malinovski, and D. J. Tannor, Phys. Rev. A 60, 3081 (1999).
${ }^{50}$ L. P. Yatsenko, V. I. Romanenko, B. W. Shore, and K. Bergmann, Phys. Rev. A 65, 043409 (2002).

${ }^{51}$ E. Geva, R. Kosloff, and J. L. Skinner, J. Chem. Phys. 102, 8541 (1995).

${ }^{52}$ E. Geva and R. Kosloff, J. Chem. Phys. 104, 7681 (1996).

${ }^{53}$ K. F. Everitt and J. L. Skinner, J. Chem. Phys. 110, 4467 (1999).

${ }^{54}$ F. Haake, Springer Tracts Mod. Phys. 66, 98 (1973).

${ }^{55}$ R. Alicki and K. Lendi, Quantum Dynamical Semigroups and Applications (Springer-Verlag, Berlin, 1987).

${ }^{56}$ W. H. Press, B. P. Flannery, S. A. Teukolsky, and W. T. Vetterling, $N u-$ merical Recipes (Cambridge University Press, Cambridge, 1986).

${ }^{57}$ L. Viola and S. Lloyd, Phys. Rev. A 58, 2733 (1998).

${ }^{58}$ G. S. Agarwal, Phys. Rev. A 61, 013809 (1999).

${ }^{59}$ D. Vitali and P. Tombesi, Phys. Rev. A 59, 4178 (1999).

${ }^{60}$ C. Search and P. R. Berman, Phys. Rev. Lett. 85, 2272 (2000).

${ }^{61}$ C. Meier and D. Tannor, J. Chem. Phys. 111, 3365 (1999).

${ }^{62}$ E. Geva, E. Rosenman, and D. J. Tannor, J. Chem. Phys. 113, 1380 (2000).

${ }^{63}$ S. Mukamel, Principles of Nonlinear Optical Spectroscopy (Oxford, New York, 1995).

${ }^{64}$ E. Geva and R. Kosloff, J. Chem. Phys. 96, 3054 (1992).

${ }^{65}$ E. Geva and R. Kosloff, J. Chem. Phys. 97, 4398 (1992).

${ }^{66}$ E. Geva and R. Kosloff, Phys. Rev. E 49, 3903 (1994). 